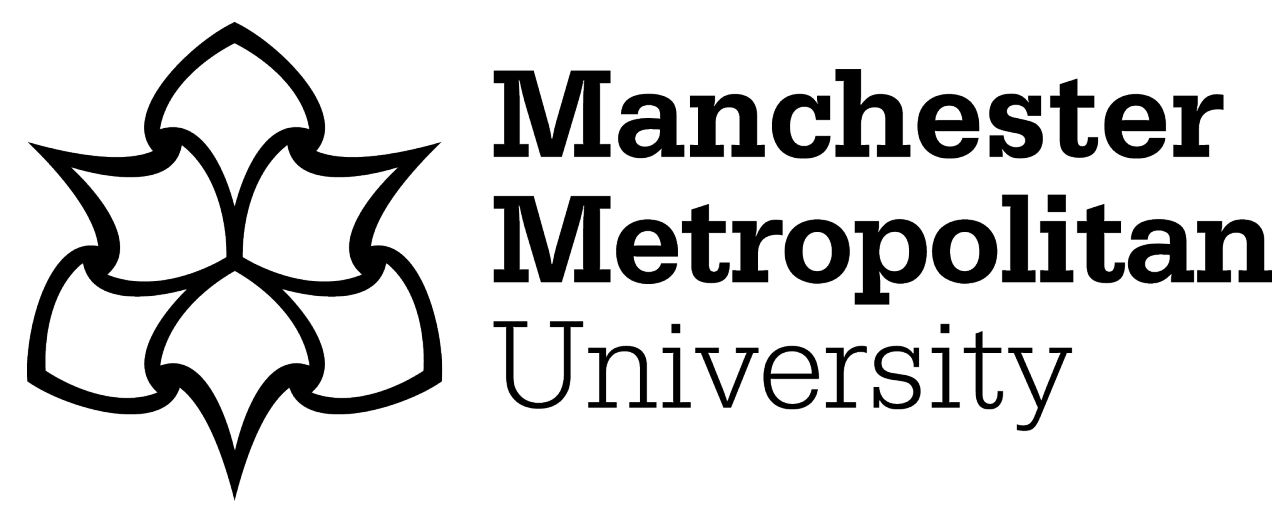

Jiang, SC, Bai, W ORCID logoORCID: https://orcid.org/0000-0002-3537207X and Tang, GQ (2018) Numerical simulation of wave resonance in the narrow gap between two non-identical boxes. Ocean Engineering, 156. pp. 38-60. ISSN 0029-8018

Downloaded from: https://e-space.mmu.ac.uk/620010/

Version: Accepted Version

Publisher: Elsevier

DOI: https://doi.org/10.1016/j.oceaneng.2018.02.055

Usage rights: Creative Commons: Attribution-Noncommercial-No Derivative Works 4.0

Please cite the published version 


\title{
Numerical simulation of wave resonance in the narrow gap between two non-identical boxes
}

\author{
Sheng-Chao Jiang ${ }^{\mathrm{a}, \mathrm{b}, *}$, Wei Baic ${ }^{\mathrm{c}}$, Guo-Qiang Tang ${ }^{\mathrm{d}}$ \\ ${ }^{a}$ School of Naval Architecture, State Key Laboratory of Structural Analysis for Industrial Equipment, Dalian University of \\ Technology, Dalian 116024, China \\ ${ }^{b}$ Collaborative Innovation Center for Advanced Ship and Deep-Sea Exploration, Shanghai 200240, China \\ ${ }^{c}$ School of Computing, Mathematics and Digital Technology, Manchester Metropolitan University, Chester Street, Manchester \\ M1 $5 G D, U K$ \\ ${ }^{d}$ State Key Laboratory of Coastal and Offshore Engineering, Dalian University of Technology, Dalian 116024, China
}

\begin{abstract}
Wave resonance in the narrow gap between two side-by-side non-identical boxes is investigated by employing a two-dimensional numerical wave flume based on the OpenFOAM ${ }^{\circledR}$ package. The focus of this study is to examine the influence of the energy transformation and the energy dissipation on the hydrodynamic behavior of wave response around resonant conditions. Numerical simulations show that the unrealistic wave resonant responses in the narrow gap by the linear potential flow model are due to not only the energy dissipation induced by the fluid rotational motion, but also the energy transformation associated with the free surface. With the increase of incident wave amplitude, relatively more energy is reflected, leading to the decrease of wave resonant response and energy dissipation in the narrow gap at the resonant frequency. When slightly away from the resonant frequency, the energy dissipation becomes the dominant factor for the decrease of wave response in the narrow gap with increasing the incident wave amplitude. As for the influence of gap configuration, on one hand, energy dissipation has the dominant effect for the typical case of small upstream and large downstream box drafts. On the other hand, the reflected energy is more important for the typical large upstream and small downstream box drafts. More resonant fluid exists in the gap with the increase of gap breadth, leading to the decrease of reflection coefficient and the increase of transmission coefficient.
\end{abstract}

Keywords: Wave resonance, Narrow gap, Energy dissipation, Energy transformation, Non-identical boxes, OpenFOAM ${ }^{\circledR}$

\section{Introduction}

2 In recent years, as the offshore oil and gas explorations and operations have moved towards deeper 3 waters and harsher environments, Floating Production Storage and Offloading (FPSO) and Floating Liquefied

4 Natural Gas (FLNG) production systems become more attractive. These structures are maintained stationary 5 by a spread or turret mooring system, and a Liquefied Natural Gas (LNG) ship or shuttle tanker periodically

\footnotetext{
${ }^{*}$ Corresponding author

Email address: jiangshengchao@foxmail.com (Sheng-Chao Jiang)
} 
approaches them for loading gas or oil according to a close proximity in side-by-side arrangement. For this loading operation, one of the key technical challenges is the fluid resonance in the narrow gap between them under the wave action.

Wave resonance in the narrow gap between two bodies in a close proximity has been studied extensively. Early examinations were focused on the theoretical study of eigenfrequency and eigenfunction of the resonant modes, based on the linearized potential flow theory. An analytical solution was derived in Molin (2001) for the barges with infinite length and beam in the infinite water depth, where the formula for the resonant frequencies of piston- and sloshing modes were obtained via solving an eigenvalue equation. Molin et al. (2002) further extended the work to the gap resonance in an open-ended narrow gap. Furthermore, Faltinsen et al. (2007) proposed an analytical method based on the domain decomposition approach, in which the piston-like mode in a two-dimensional moonpool between two heaving rectangular floating hulls in the finite water depth was discussed. Besides the analytical solutions, numerical simulations in the framework of potential flow theory have also been adopted to investigate the resonant modes, for example in Sun et al. (2010) where the free surface piston- and sloshing-modal resonant behaviour around two adjacent barges was investigated by using the second-order potential flow analysis in the frequency domain.

According to extensive comparisons, it has been demonstrated that the potential flow model is capable of predicting the resonant frequencies and capturing the resonant modes. However, the potential flow model was reported to over-predict the resonant amplitudes. Focused on this problem, Saitoh et al. (2006) conducted a set of experimental investigations in a wave flume, and suggested that the resonant amplitudes are dependent on the body draft and gap breadth. This conclusion is consistent with the analytical and experimental results of Molin (2001). Iwata et al. (2007) extended this work to the three-body problem, indicating that the number of boxes also has the significant effect on resonant phenomena. At the same time, with the fast development of computing technology and numerical technique, Computational Fluid Dynamics (CFD) simulation has also been taken as an alternative method in recent years. Lu, Teng, Cheng, Sun and Chen (2011); Lu, Teng, Sun and Chen (2011) investigated the variations of resonant amplitudes by a three-step high-order upwind Taylor-Galerkin Finite Element Method (FEM). In Moradi et al. (2016) the effect of water depth on resonant behavior of the fluid trapped between two side-by-side bodies was studied. Numerical results found that the potential flow model not only over-predicts the resonant wave amplitude, but also gives an incorrect variation tendency of the resonant wave amplitude with water depth.

In order to reduce the computational cost of fully CFD simulations, the coupling model based on the domain decompositions method has been also established. Elie et al. (2013) adopted the approach of Spectral Wave Explicit Navier-Stokes Equations (SWENSE), which is a combination of the linear potential flow model in the frequency domain and the viscous fluid model with RANS turbulent equations, to simulate the gap resonance between side-by-side barges. In addition, in the numerical simulation of Fredriksen et al. (2014), as well as the early work in Kristiansen and Faltinsen (2012), the coupling models based on the domain decomposition were adopted, in which the laminar Navier-Stokes equations were applied in the lower region 
of the gap, while the potential flow model was used in the upper region of the gap and the outer region. This in fact acquiesces that the fluid rotational motion is important around the gap bottom, while the potential model may be enough for the free surface simulation. Generally, acceptable results of resonant amplitude can be obtained in various experimental tests and CFD simulations, however, the mechanical essence behind the hydrodynamic behavior of the resonant phenomena is still an interesting field. On one hand, it is an important academic problem on the topic of wave and multi-body interactions, which is one type of socalled 'trapped structures' in a broad sense. On the other hand, an essential understanding about the major factor on the over-prediction by potential flow models can help to develop an approximated method for the convenient use in industry.

It seems to be speculated that the over-predictions of wave resonance come from the ignorance of the inherent fluid viscosity in potential flow models. Based on this hypothesis, attempts to introduce some damping artificially in the linear potential flow model has been suggested. Newman (2004) modelled a damping term as the body force on the free surface between side-by-side vessels, and Chen (2004) introduced a damping force term into the free surface boundary conditions, which was explained as energy dissipation. The efficiency of the linear dissipative term was presented by Jean-Robert et al. (2006) with comparisons to the commercial software WAMIT ${ }^{\circledR}$ and HydroStar ${ }^{\circledR}$, as well as measured data. These modified potential flow models are able to suppress unrealistic values, but still cannot capture the actual physical sense. A CFD simulation by Lu et al. (2010) suggested that the wave amplitude in the narrow gap is closely relevant to the vertical velocity along the gap bottom. Examinations of flow pattern indicated that the most violent rotational flow field happens in the vicinity of the gap entrance, where the significant vortex shedding and attached vortex structure can be observed. Faltinsen and Timokha (2015) accounted for the vortex-induced damping by quantifying a pressure discharge condition in the gap opening. The calculations can be supported by their earlier experimental and numerical data in Faltinsen et al. (2007). Lu and Chen (2012) quantitatively calculated the energy dissipation rate for the fluid resonance in the narrow gap induced by waves based on the Navier-Stokes flow solutions. It suggested that the flow separation and vortex motion play the most important role in energy dissipation for a wall bounded region. The majority of energy dissipation happens around the gap entrance, not on the free surface in the narrow gap. Similar findings were also obtained by Kristiansen and Faltinsen (2010) for the piston-mode wave resonance in the gap formed by a ship model arranged in front of a vertical wall. However, due to the existence of vortices square term in the expression of energy dissipation rate, it is not easy to compute the energy dissipation directly, and a clear relationship between energy dissipation and resonant amplitude or other parameters is not currently available.

In addition to the energy dissipation by the fluid viscosity, the process of energy transformation due to the large-amplitude free surface motion may also play an important role on wave resonances in the narrow gap. A fully nonlinear potential flow model was adopted by Feng and Bai 2015) for the wave resonance between two barges, in which the lateral piston mode and longitudinal sloshing mode were successfully captured. In their study, although the free surface nonlinearity was found to play a minor role in suppressing the over- 
predicted resonance response, nonlinear analysis illustrated the gap resonance to be equivalent to a stiff spring in a nonlinear mass-spring system: the resonant frequency slightly shifts to higher values as incident wave steepness increases. This is an important process of energy transformation due to the large-amplitude pistonlike free surface oscillation. Ananthakrishnan (2015) investigated the effect of viscosity and nonlinearity on the forces and waves generated by a floating twin hulls under heave oscillations. Numerical results showed that the nonlinear effect on the wave forces is significant at all frequencies for the large amplitude oscillation relative to the hull draft. Besides, the influence of interaction between the energy transformation in the free surface motion and the energy dissipation in the fluid rotational motion can be speculated during the process of wave resonance. It might be important for understanding the essential hydrodynamic behavior of the fluid resonance in the narrow gap.

The motivation of this study is to investigate the influence of energy transformation and energy dissipation on the hydrodynamic behavior of wave responses around the resonant frequency. In the previous studies, all floating objects were modelled as the identical bodies, whereas in reality the objects may have different sizes, such as the most typical loading or offloading operations of the side-by-side arrangement between FPSO and LNG vessels. Therefore, the system with two non-identical boxes is taken as the background of this study, as the non-identical nature of the system may affect both the resonant frequency and amplitude in the narrow gap compared to the identical box systems. Besides the wave amplitude in the narrow gap, the reflection and transmission coefficients are also attributed to the process of energy transformation. Moreover, the quadratic sum of the reflection and transmission coefficients, $\mathbb{E}=K_{r}^{2}+K_{t}^{2}$, defined as energy coefficient, is adopted for examining the energy dissipation. Under the framework of linear potential flow theory, the principle of energy conservation ensures the energy coefficient keeps at $\mathbb{E}=1$. The value of $\mathbb{E}$ by the present viscous flow model can give us a new view on the energy dissipation due to the influence of fluid viscosity. In sum, an integral comprehensive understanding on the mechanical essence of the gap resonance is expected from the perspective of energy transformation and energy dissipation in the current study.

In Sections 2, 3 and 4, the numerical wave flume used in this work is presented, setup and validated against available experimental and numerical data, respectively. The numerical results and discussions are presented in Section 5 to show the effect of energy transformation and energy dissipation on wave responses around the resonant frequency, including the comparisons of results between the linear potential flow model and the present CFD model, and the influence of gap configuration and incident wave amplitude. Finally, conclusions are drawn in Section 6 .

\section{Mathematical Formulation}

The governing equations for the mass and momentum conservations in an Eulerian reference system for incompressible two-phase flows can be given as,

$$
\frac{\partial \rho u_{i}}{\partial x_{i}}=0,
$$


111

$$
\frac{\partial \rho u_{i}}{\partial t}+\frac{\partial \rho u_{i} u_{j}}{\partial x_{j}}=\rho g_{i}-\frac{\partial p}{\partial x_{i}}+\mu \frac{\partial}{\partial x_{j}}\left(\frac{\partial u_{i}}{\partial x_{j}}+\frac{\partial u_{j}}{\partial x_{i}}\right),
$$

where $u_{i}$ is the velocity component in the $i$ th direction, and $u_{i}^{m}$ is the velocity component due to the mesh deformation in the ALE frame. $p, \rho$ and $g_{i}$ are the pressure, fluid density and gravitational acceleration, respectively, and $\mu$ is the fluid dynamic viscosity.

In this study, the Volume of Fluid (VOF) method (Hirt and Nichols, 1981), documented by Berberović et al. (2009), is adopted to capture the free surface motion. The fractional function of VOF, defined by $\varphi$, in a computational cell is defined as,

$$
\varphi= \begin{cases}0, & \text { in air } \\ 0<\varphi<1, & \text { on free surface } \\ 1, & \text { in water }\end{cases}
$$

The VOF function satisfies the following advection equation,

$$
\frac{\partial \varphi}{\partial t}+u_{i} \frac{\partial \varphi}{\partial x_{i}}=0 .
$$

Herein, the contour of VOF function with $\varphi=0.5$ is used to represent the interface between the water and air phases. In the computations, the fluid density and effective viscosity are averaged by using the available VOF function,

$$
\rho=\varphi \rho_{W}+(1-\varphi) \rho_{A},
$$

$$
\mu=\varphi \mu_{W}+(1-\varphi) \mu_{A},
$$

where the subscripts $W$ and $A$ represent the Water phase and Air phase, respectively.

In the present numerical wave flume, relaxation zones are adopted to generate the incident wave and eliminate the transmission wave at the inlet and outlet boundaries, respectively. Moreover, it can also be implemented to avoid the internal wave reflection in the computational domain. A relaxation function

$$
\alpha_{R}\left(\chi_{R}\right)=1-\frac{\exp \left(\chi_{R}^{3.5}\right)}{\exp (1)-1} \quad \chi_{R} \in[0,1]
$$

is applied inside the relaxation zone in the following way,

$$
\vartheta=\alpha_{R} \vartheta_{C}+\left(1-\alpha_{R}\right) \vartheta_{T},
$$

where $\vartheta$ is either $u_{i}$ or $\varphi$, and the subscripts $C$ and $T$ represent the Computed value and Target value, respectively. The variation of $\alpha_{R}$ is the same as in Fuhrman et al. (2006), where $\alpha_{R}$ in Eq. (5) is only activate in the relaxation zone, and it is always 1 at the interface between the non-relaxed part of the computational domain. Detailed information about the relaxation technique can be found in Mayer et al. (1998), Engsig-Karup (2006) and Jacobsen et al. (2012). 
The governing equations (1a)- $1 \mathrm{~b}$ ) and the VOF equation (3) are solved based on the Finite Volume Method (FVM) integrated in the OpenFOAM ${ }^{\circledR}$ package. The velocity and pressure are decoupled by the Pressure Implicit with Splitting of Operators algorithm (PISO) (Issa, 1986). The Euler method is used to discretize the transient term. The convection term and diffusion term are discretized by the Gauss Limited Linear method and the Gauss Linear Corrected method, respectively. The numerical computations always start from the still state, which means the hydrostatic pressure and zero velocity are specified as the initial conditions. The no-slip boundary condition is imposed at the solid wall including the body surface and seabed. At the upper boundary of the numerical wave flume, a reference pressure $p=0$ and a velocity condition $\frac{\partial \mathbf{u}}{\partial \mathbf{n}}$ are implemented with $\mathbf{n}$ the outward unit normal vector. The interface tension between the air and water phases is neglected in this study since the dynamic effects from the air phase are very small. At the two ends of the spongy layer, zero velocities are applied considering that the waves are damped out there by the spongy layer. For the details of numerical implements in OpenFOAM ${ }^{\circledR}$, the readers may refer to Jasak (1996) and Rusche (2003).

In the present numerical simulation, the time increment is automatically determined according to the Courant-Friedrichs-Lewy (CFL) condition,

$$
\Delta t \leq C_{r} \times \min \left\{\sqrt{S_{e}} /\left|u_{e}\right|\right\}
$$

where $S_{e}$ and $\left|u_{e}\right|$ are the area and absolute velocity in a computational cell, respectively. The numerical experience in the present study confirms that the coefficient $C_{r}=0.2$ can produce stable and accurate results. It should be mentioned that the classical linear potential flow model is also adopted in this study for the purpose of comparison, for which the theoretical formulation is omitted here, as it is well known in many textbooks.

\section{Numerical Setup}

The definition sketch of the present numerical simulations is illustrated in Fig. 11 in which the origin of the coordinate system is located at the still water level and the wave is propagating in the positive $x$ direction. Two boxes, defined as Box $\mathrm{A}$ and Box $\mathrm{B}$, with the identical breadth $B=0.50 \mathrm{~m}$ but different drafts $D_{L}$ and $D_{R}$ for the upstream (left) and downstream (right) boxes, respectively, are fixed in a wave flume with the water depth $h=0.50 \mathrm{~m}$. A narrow gap with the breadth $B_{g}$ is formed by the two boxes, where the extremely large amplitude of fluid resonance can be excited as the incident wave frequency is close to the natural frequency of the confined fluid bulk. A number of simulation cases are designed to perform the intended investigation by varying the values of gap breadth $B_{g}$, and upstream and downstream box drafts $D_{L}$ and $D_{R}$. The definition of the test cases and the corresponding configurations of box drafts $D_{L}$ and $D_{R}$ are tabulated in Tab. 1. Four gap breadths $B_{g}=0.030 \mathrm{~m}, 0.050 \mathrm{~m}, 0.070 \mathrm{~m}, 0.090 \mathrm{~m}$ and three incident wave amplitudes $A_{i}=0.008 \mathrm{~m}, 0.012 \mathrm{~m}$ and $0.016 \mathrm{~m}$ are selected, so totally 192 different cases are considered. In the following descriptions, the prefix 'Bg' would be adopted for identifying the gap breadth, for example 


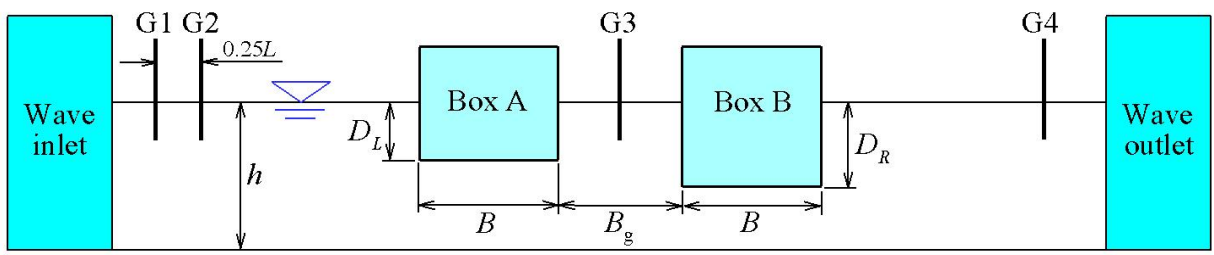

Figure 1: Definition sketch of the numerical wave flume based on the resonant frequency of fluid in the narrow gap, which is the extension of those used in Saitoh et al. (2006), Lu et al. (2010) and Moradi et al. (2016).

Bg50DL103DR205 means $B_{g}=0.050 \mathrm{~m}, D_{L}=0.103 \mathrm{~m}$ and $D_{R}=0.252 \mathrm{~m}$. The wave frequency $\omega$ is chosen

Table 1: List of test cases and corresponding configurations in the present study

\begin{tabular}{lllll}
\hline & $D_{R}=0.103 \mathrm{~m}$ & $D_{R}=0.153 \mathrm{~m}$ & $D_{R}=0.202 \mathrm{~m}$ & $D_{R}=0.252 \mathrm{~m}$ \\
\hline$D_{L}=0.103 \mathrm{~m}$ & DL103DR103 & DL103DR153 & DL103DR202 & DL103DR252 \\
$D_{L}=0.153 \mathrm{~m}$ & DL153DR103 & DL153DR153 & DL153DR202 & DL153DR252 \\
$D_{L}=0.202 \mathrm{~m}$ & DL202DR103 & DL202DR153 & DL202DR202 & DL202DR252 \\
$D_{L}=0.252 \mathrm{~m}$ & DL252DR103 & DL252DR153 & DL252DR202 & DL252DR252 \\
\hline
\end{tabular}

The height of the numerical wave flume is fixed at $0.8 \mathrm{~m}$, and the length is closely relevant to the incident wave length $L$ for different simulations. In numerical simulations, two relaxation zones are arranged on the left and right sides of the wave flume, respectively. Generally, the length of the relaxation zone is around 1.5-2.0L. As shown in Fig. 1] four wave gauges, G1-G4, are equipped to record the wave elevation. G1 and G2 are used for separating the incident and reflected waves, in which the distance between them is kept at $0.25 L$, while G3 and G4 are used to record the wave response in the narrow gap and the transmission wave, respectively. G3, G2 and G4 are situated in the middle of the narrow gap, 1.5L from the left side of Box A, and $1.5 L$ from the right side of Box B, respectively. In addition, it is noted that we don't restrict $D_{L}<D_{R}$ in this study.

\section{Numerical Validation}

The mesh resolution tests are carried out first by using four different meshes for two kinds of structures, Bg30DL103DR103 and Bg70DL252DR252 in Tab.11. Tab.2 2illustrates the detailed mesh information. Herein, non-uniform meshes are adopted for saving the computational time. The square fine meshes with high resolution are adopted around the boxes, especially in the vicinity of the narrow gap, to accurately capture the large-amplitude free surface oscillation and to account for the boundary layer effect. In the relaxation zone of eliminating the transmission wave, coarse rectangular meshes with large aspect ratio up to $1 / 20$ 


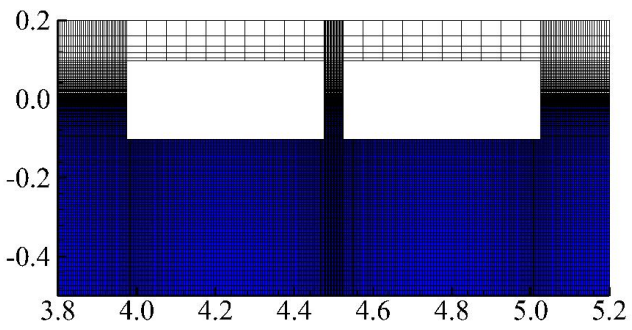

(a) $B_{g}=0.030 \mathrm{~m}, D_{L}=D_{R}=0.103 \mathrm{~m}$

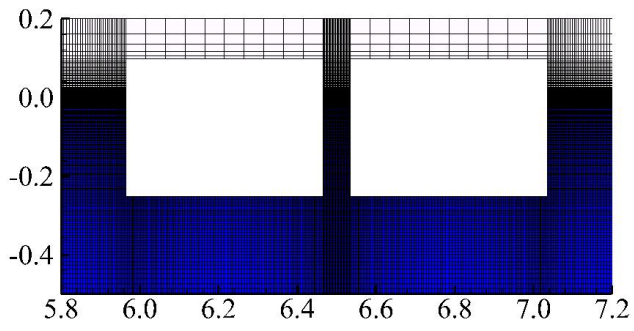

(b) $B_{g}=0.070 \mathrm{~m}, D_{L}=D_{R}=0.252 \mathrm{~m}$

Figure 2: Typical computational meshes in the vicinity of the narrow gap

Table 2: Mesh information for convergent tests (Elements/Nodes)

\begin{tabular}{cccccc}
\hline$B_{g}(\mathrm{~m})$ & $D_{L}=D_{R}(\mathrm{~m})$ & Mesh 1 & Mesh 2 & Mesh 3 & Mesh 4 \\
\hline 0.030 & 0.103 & $96100 / 194418$ & $130052 / 262726$ & $170400 / 343888$ & $212375 / 428068$ \\
0.070 & 0.252 & $100896 / 204180$ & $131985 / 266736$ & $162282 / 327676$ & $221737 / 447056$ \\
\hline
\end{tabular}

According to the linear potential flow analysis, the resonant frequencies of fluid oscillation in the narrow gap can be estimated as 8.00 and $5.10 \mathrm{rad} / \mathrm{s}$ for the cases mentioned above, respectively. They are adopted as the incident wave frequency for the mesh resolution tests, in which the incident wave amplitude $A_{i}=0.012$ $\mathrm{m}$ is considered. The time signals of wave oscillation in the narrow gap measured at Probe G3 during 40 - 50 seconds with various mesh resolutions are compared in Fig. 3. A steady state of large-amplitude piston-like wave elevations can be observed clearly, implying that the relaxation zones can work well in eliminating the reflection and transmission waves. Very little discrepancy between Mesh 3 and Mesh 4 can be observed in this figure, which indicate that the convergent solutions can be produced by Mesh 3 for various structures. Numerical simulations also suggest that the measured steady-state free surface evolution is quite symmetrical and sinusoidal, and the dominating harmonic oscillates at the incident wave frequency $\omega$ according to the Fourier analysis. Furthermore, the normalized wave amplitudes $A_{g} / A_{i}$ in the narrow gap at the Probe G3 are compared in Fig. 4 It should be mentioned that the wave amplitudes $A_{g}$ in the narrow gap are computed by the averaged value of wave amplitudes between the duration of 40 - 60 seconds, after the sensitivity analysis by considering different time-windows of 60 - 80 seconds. The comparisons in Fig. 4 suggest that the variation of mesh density has little effect on the present numerical results if the number of cells exceeds $1.6 \times 10^{5}$. Again, Mesh 3 is able to produce convergent solutions, and hence it is adopted as the baseline for 
the following numerical computations.

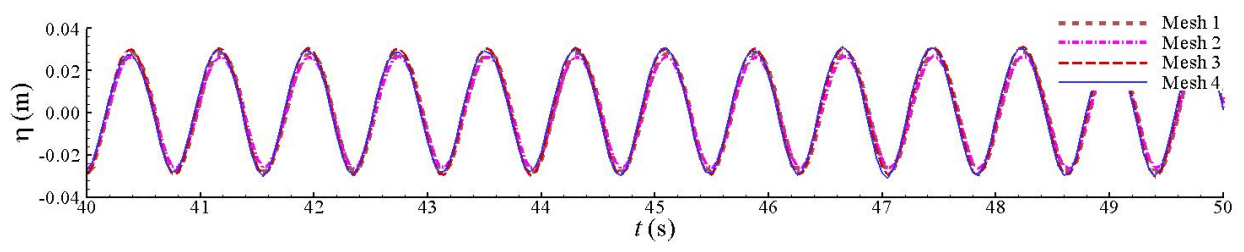

(a) $B_{g}=0.030 \mathrm{~m}, D_{L}=D_{R}=0.103 \mathrm{~m}$

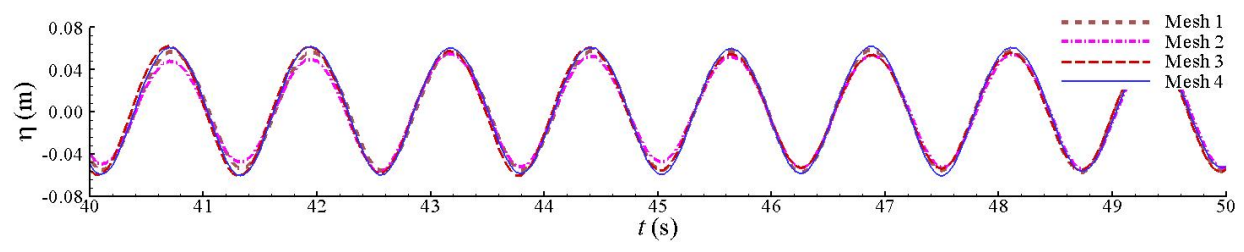

(b) $B_{g}=0.070 \mathrm{~m}, D_{L}=D_{R}=0.252 \mathrm{~m}$

Figure 3: Comparisons of wave elevation in the gap under different meshes with various box drafts $D$ at the resonant frequency $\omega_{g}$

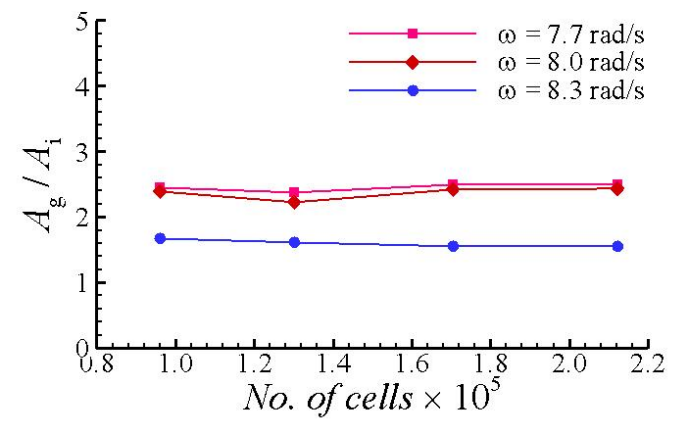

(a) $B_{g}=0.030 \mathrm{~m}, D_{L}=D_{R}=0.103 \mathrm{~m}$

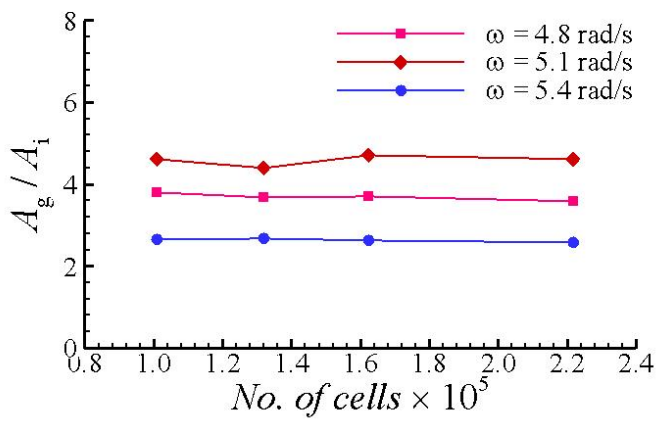

(b) $B_{g}=0.070 \mathrm{~m}, D_{L}=D_{R}=0.252 \mathrm{~m}$

Figure 4: Mesh convergent test for various narrow gap configurations with different incident wave frequencies $\omega$ around the resonant frequency $\omega_{g}$

The numerical accuracy of the present numerical model is validated by comparing with the available laboratory test results in Saitoh et al. (2006) and the numerical results in Lu, Teng, Cheng, Sun and Chen (2011). In their study, only the identical boxes of $D_{L}=D_{R}$ are examined, and hence we denote the drafts $D_{L}$ and $D_{R}$ as $D$ for simplification in this section. The mean normalized wave amplitude $A_{g} / A_{i}$ at G3 (located in the center of the narrow gap) is compared in Fig. 5 , in which the incident wave amplitude is fixed at $A_{i}$ $=0.012 \mathrm{~m}$ and measured by G1 and G2 wave gauges. Again, the averaged wave amplitudes in the steady state between 40 - 60 seconds are computed as $A_{g}$. Generally speaking, the present numerical results are in agreement with both the experimental measurements and the numerical solutions. All these comparisons 


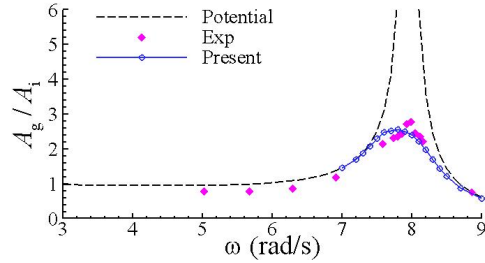

(a) $B_{g}=0.030 \mathrm{~m}, D=0.103 \mathrm{~m}$

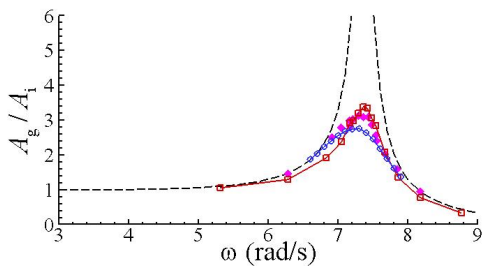

(d) $B_{g}=0.050 \mathrm{~m}, D=0.103 \mathrm{~m}$

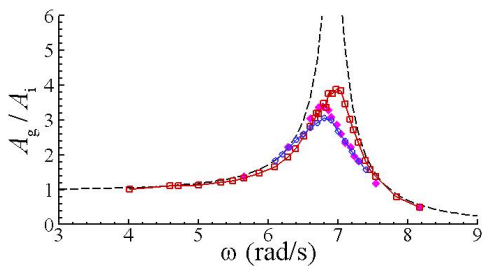

(g) $B_{g}=0.070 \mathrm{~m}, D=0.103 \mathrm{~m}$

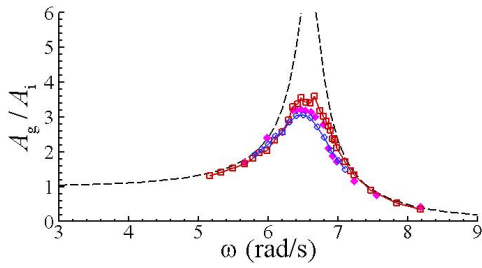

(j) $B_{g}=0.090 \mathrm{~m}, D=0.103 \mathrm{~m}$

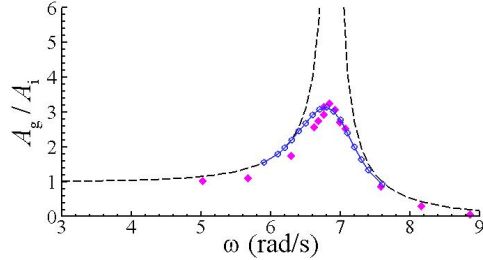

(b) $B_{g}=0.030 \mathrm{~m}, D=0.153 \mathrm{~m}$

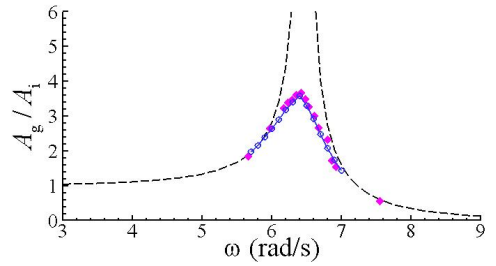

(e) $B_{g}=0.050 \mathrm{~m}, D=0.153 \mathrm{~m}$

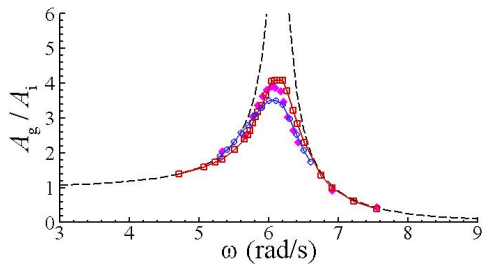

(h) $B_{g}=0.070 \mathrm{~m}, D=0.153 \mathrm{~m}$

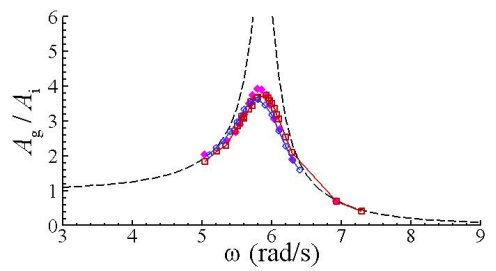

(k) $B_{g}=0.090 \mathrm{~m}, D=0.153 \mathrm{~m}$

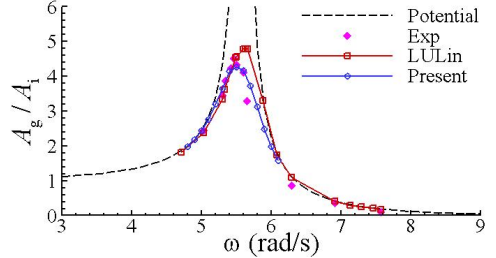

(c) $B_{g}=0.030 \mathrm{~m}, D=0.252 \mathrm{~m}$

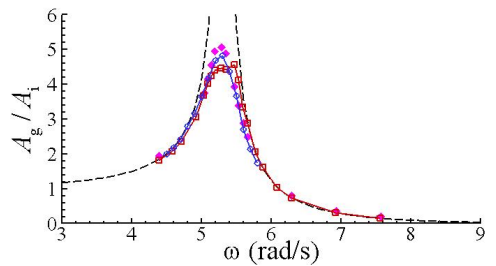

(f) $B_{g}=0.050 \mathrm{~m}, D=0.252 \mathrm{~m}$

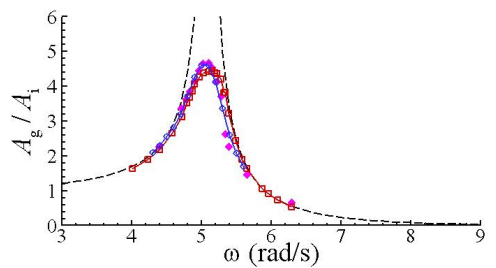

(i) $B_{g}=0.070 \mathrm{~m}, D=0.252 \mathrm{~m}$

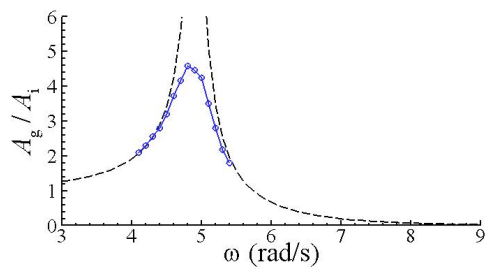

(l) $B_{g}=0.090 \mathrm{~m}, D=0.252 \mathrm{~m}$

Figure 5: Comparison of normalized wave amplitude $A_{g} / A_{i}$ for various gap breadths and drafts

Validations are also extended to the results of reflection and transmission coefficients in this work. The reflection and transmission coefficients are defined as $K_{r}=A_{r} / A_{i}$ and $K_{t}=A_{t} / A_{i}$, respectively, where $A_{r}$ and $A_{t}$ are the reflected and transmitted wave amplitudes, measured at Probes G1, G2 and G4. In accordance with the wave amplitudes in the narrow gap, $A_{r}$ and $A_{t}$ are also the averaged wave amplitudes in the steady 
state between 40 - 60 seconds. Fig. 6 depicts the comparisons of reflection and transmission coefficients, $K_{r}$ and $K_{t}$, for Bg50DL252DR252 between the present numerical results, the measured data in Saitoh (2007) and the numerical results in Lu et al. (2010). In addition, for the purpose of comparison, results by the potential flow model are also presented in this figure. Fig. 6a shows that for the reflection coefficient, the frequency of the minimal peak value predicted by the viscous fluid models and potential flow model are almost identical to the observation in the experiments, which is the resonant frequency of fluid oscillation in the narrow gap. The variations of $K_{r}$ against $\omega$ predicted by the two viscous models are found to be in good agreement with the experimental data. Whereas, the potential flow model gives a significant minimal peak value at the resonant frequency. The discrepancies between the two sets of viscous fluid numerical results at the high wave frequencies might be due to the different numerical models adopted. The numerical model adopted in Lu et al. (2010) is the three-step high-order upwind Taylor-Galerkin FEM, where the issue of numerical dissipation might need more attention. As the increase of incident wave frequency, the increasing wave steepness is expected, leading to more numerical dissipation in the numerical simulations, especially in the region of high wave frequency in Fig,6a As for the predictions of transmission coefficient in Fig. 6b, the maximal value of transmission coefficient predicted by the potential flow model is significantly larger than that in the experimental tests and by the viscous fluid models.

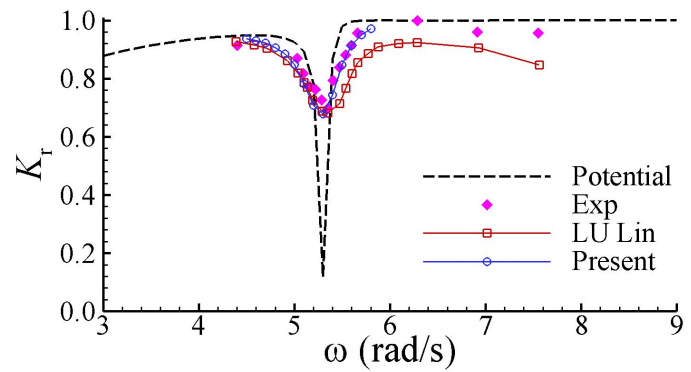

(a) Reflection coefficient $K_{r}$

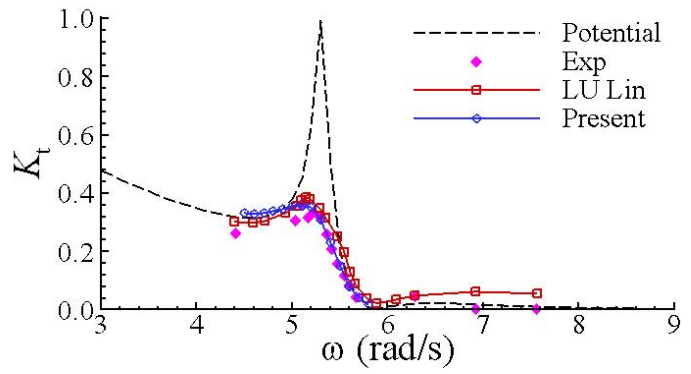

(b) Transmission coefficient $K_{t}$

Figure 6: Comparison of reflection and transmission coefficients for the case of $B_{g}=0.005 \mathrm{~m}, D_{L}=0.252 \mathrm{~m}$ and $D_{R}=0.252$ $\mathrm{m}$

The comparisons shown in Figs. 5 and 6 confirm that the present numerical wave flume works well, and is capable of producing numerical results in good agreement with the experimental data both on the wave amplitude in the narrow gap and the reflection and transmission coefficients. However, the potential flow model may over-predict the resonant response in the narrow gap and the minimal and maximal values of the reflection and transmission coefficients.

\section{Numerical Results and Analysis}

The validation study in the previous section shows that the present numerical wave flume is able to reproduce well the studied scenario of gap resonance between two rectangular boxes. It is employed to 
investigate the fluid resonance in the narrow gap formed by two non-identical boxes under wave actions. As mentioned above, in total 192 cases listed in Tab. 1 are considered herein. In order to demonstrate the necessity of adopting the viscous fluid model for this problem, the linear potential flow solutions are also included for the purpose of comparison. Numerical results include the resonant wave amplitude in the narrow gap, the reflection and transmission coefficients of the two-box system, and the quadratic sum of reflection and transmission coefficients, $\mathbb{E}=K_{r}^{2}+K_{t}^{2}$, defined as energy coefficient for examining the energy dissipation. Again, all these parameters are computed by the averaged values in the steady state between 40 - 60 seconds.

\subsection{General description of wave response}

Numerical investigations begin with the variation of wave response in the narrow gap against the incident wave frequency with the incident wave amplitude $A_{i}=0.012 \mathrm{~m}$, for the cases of $B_{g}=0.050 \mathrm{~m}$, as shown in Fig. 7. It is found that the resonant frequency predicted by the two numerical models is almost identical for each gap configuration considered in this work. However, the significant over-predicted resonant wave amplitudes in the narrow gap can be observed in the potential flow results. The major reason for the over-prediction is that the potential flow theory, in which the fluid is assumed to be inviscid and the flow irrotational, cannot model the influence of the fluid viscosity correctly. On the other hand, the potential flow model can work well if the incident wave frequencies are outside the range of resonant frequencies. At these frequencies away from the resonant frequency, the relatively small wave response in the narrow gap can be observed, which corresponds to the small wave amplitude on the gap surface and the slow rotational flow in the vicinity of the narrow gap. Therefore, the influence of the eddying motion and energy dissipation is negligible.

Further examinations on the hydrodynamic behavior of the fluid resonant phenomena are extended to the reflection and transmission coefficients, $K_{r}$ and $K_{t}$, including the results of potential flow and viscous fluid flow models. As shown in Fig. 8 , the potential flow model can firstly manifest a general impression of the variation of $K_{r}$ and $K_{t}$ with incident wave frequencies. When the incident wave frequency tends to zero, $K_{r}$ and $K_{t}$ approach to 0 and 1, respectively, implying the total transmission happens. Oppositely, the total reflection phenomenon can be observed when the incident wave frequency gives rise to infinite, leading to the results of $K_{r}$ and $K_{t}$ to be 1 and 0 , respectively. This is a typical behavior of the two-dimensional wave-body interaction problem, and the effect of wave length is the main reason, which can be simulated by the potential flow model, correctly. However, the potential flow model cannot predict an accurate results of reflection and transmission coefficients around resonant frequencies. The comparisons of transmission coefficients in Fig. 8 suggest that the results of the viscous fluid flow model are always much smaller than those of the potential flow model. This is because the transmission coefficients are closely connected to the amplitude of wave response in the narrow gap. The fluid oscillation in the narrow gap can be taken as a radiation source for the downstream wave, leading to the transmission waves. In difference to the linear potential flow analysis, the shear shedding and eddying motion due to the fluid viscosity in the narrow gap can reduce the resonant wave amplitude, and consequently lead to the smaller transmission coefficient. 

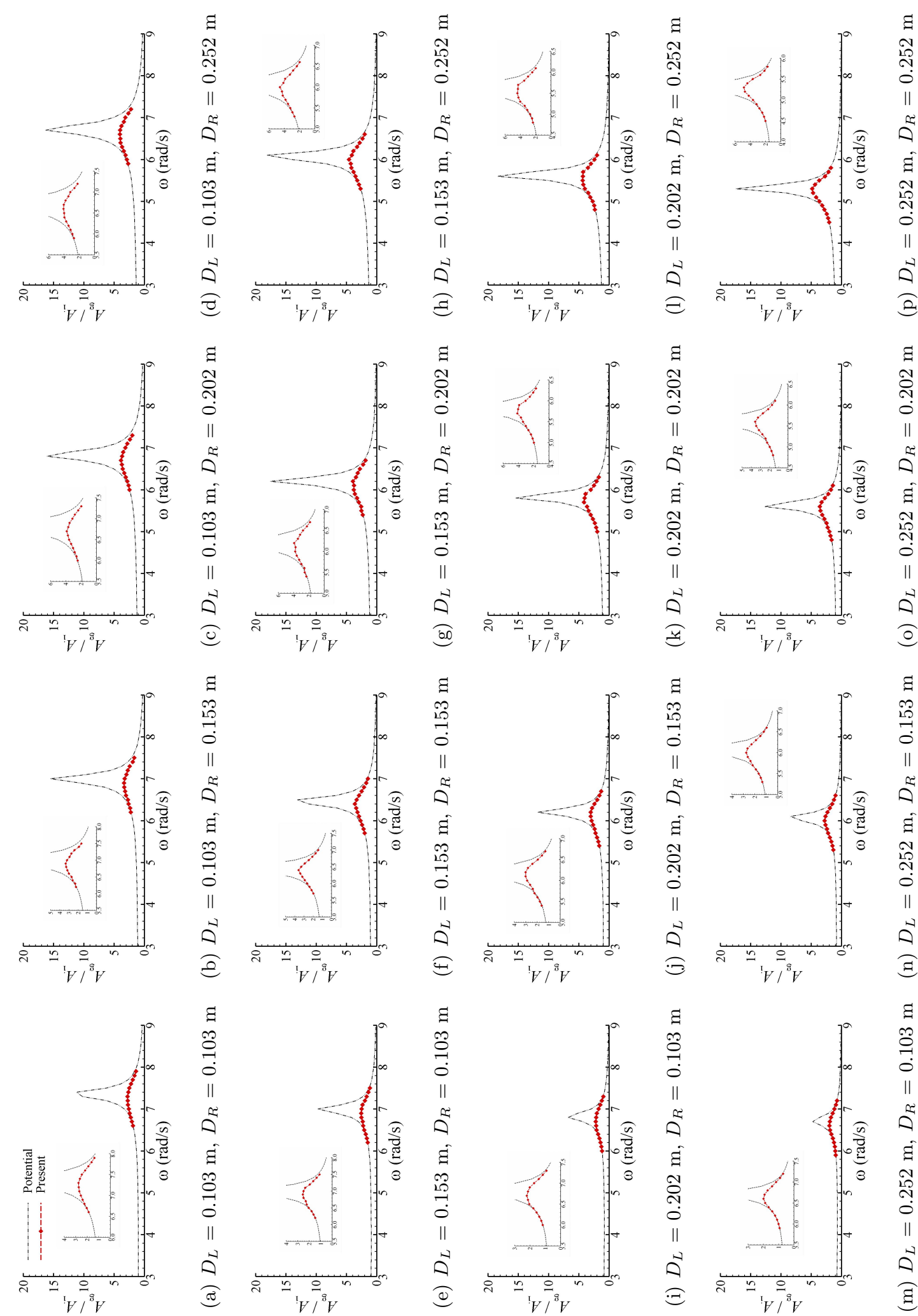

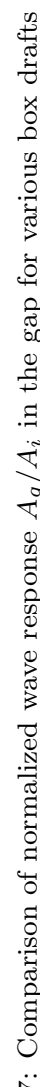

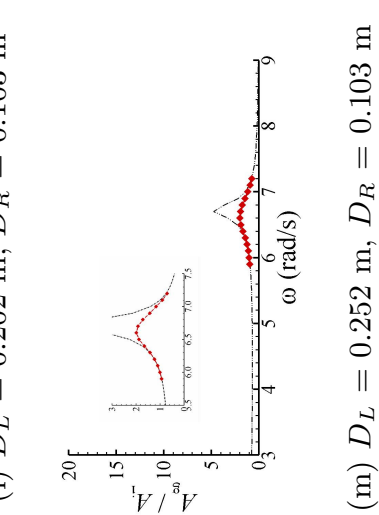



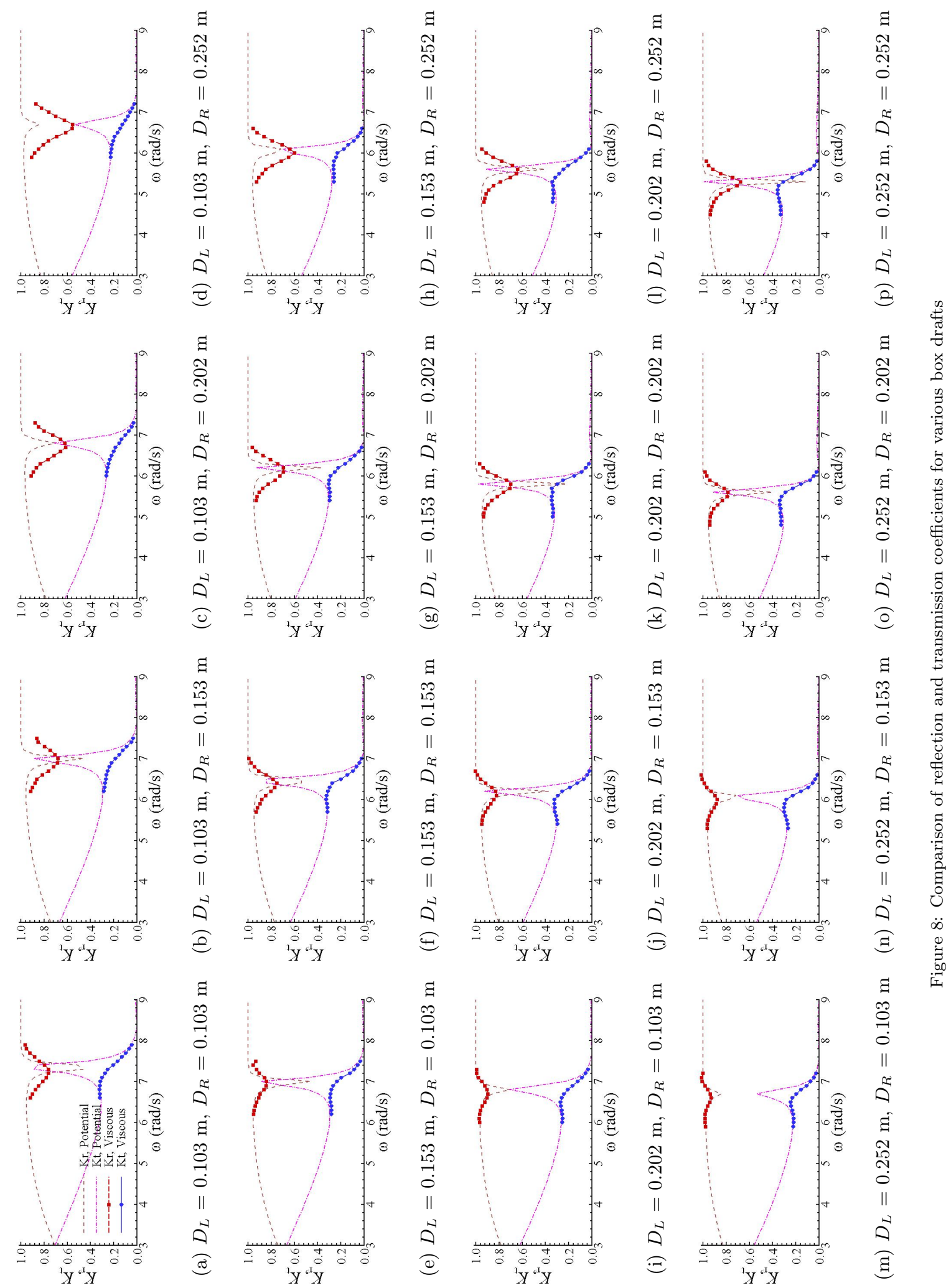
As for the reflection coefficient, there is no simple regular pattern in the discrepancy between the potential flow and viscous flow results. In Figs. $8 \mathrm{c}, 8 \mathrm{~d}$ and $8 \mathrm{~h}$, the viscous flow results are always smaller than the potential flow ones. This is the typical behavior of a system with a smaller upstream and a larger downstream box drafts. Because the narrow gap is exposed to the incident wave directly, the large-amplitude piston-mode free surface oscillation in the narrow gap can be induced, which can in turn cause the significant energy dissipation, leading to the decrease of reflection wave energy. In Figs. $8 \mathrm{i}$. $8 \mathrm{~m}$ and $8 \mathrm{n}$, the other typical behavior of a system with a larger upstream and a smaller downstream box drafts can be observed, in which the reflection coefficients by the viscous flow model are always larger than those by the potential flow model, and most of the incident wave energy is reflected by the upstream box, leading to the smaller wave response and less energy dissipation in the narrow gap. In this case, the energy dissipation in the narrow gap between two boxes is not the only dominant issue. The energy transformation due to the reflection from the upstream box also play an important role around resonant frequencies. Many factors can affect the energy transformation, such as the shielding effect of upstream box on the fluid in the narrow gap, the largeamplitude free surface oscillation in front of the two-box system, and so on. In other figures, the reflection coefficient given by the viscous flow model are larger than that of the potential flow analysis at the resonant frequency for each case. However, the viscous flow results seem to have a wider band, and become smaller when slightly away from the resonant frequency, compared to the potential flow results. It is in fact the transition between the two typical cases mentioned above. In sum, the reflection coefficient shows different hydrodynamic behaviors for various box drafts in Fig. 8, which is closely dependent on the sequence of those non-identical boxes.

Further analysis is carried out for the quadratic sum of reflection and transmission coefficients, $\mathbb{E}=$ $K_{r}^{2}+K_{t}^{2}$, the energy coefficient as defined before. The values of $\mathbb{E}$ can give us a new view on the energy dissipation. Fig. 9 shows the results of energy coefficients by both the potential flow and viscous flow models. As expected, in the framework of conventional potential flow theory, the law of energy conservation determinates the relationship of $\mathbb{E}=1$, indicating no energy is dissipated. As for the viscous flow model, a smaller energy coefficient around the resonant frequency can be seen, confirming that significant energy dissipation happens. Typical case of smaller upstream and larger downstream box drafts in Fig. 9d gives the largest energy dissipation at the resonant frequency among all the cases in Fig. 9. While the smallest energy dissipation at the resonant frequency can be found in Fig. $9 \mathrm{~m}$, which is the typical case of larger upstream and smaller downstream box drafts. Except for wave frequencies in the vicinity of the resonant frequency, the energy coefficient gives rise to $\mathbb{E}=1$, implying that the energy dissipation due to the fluid rotational motion is negligible. 

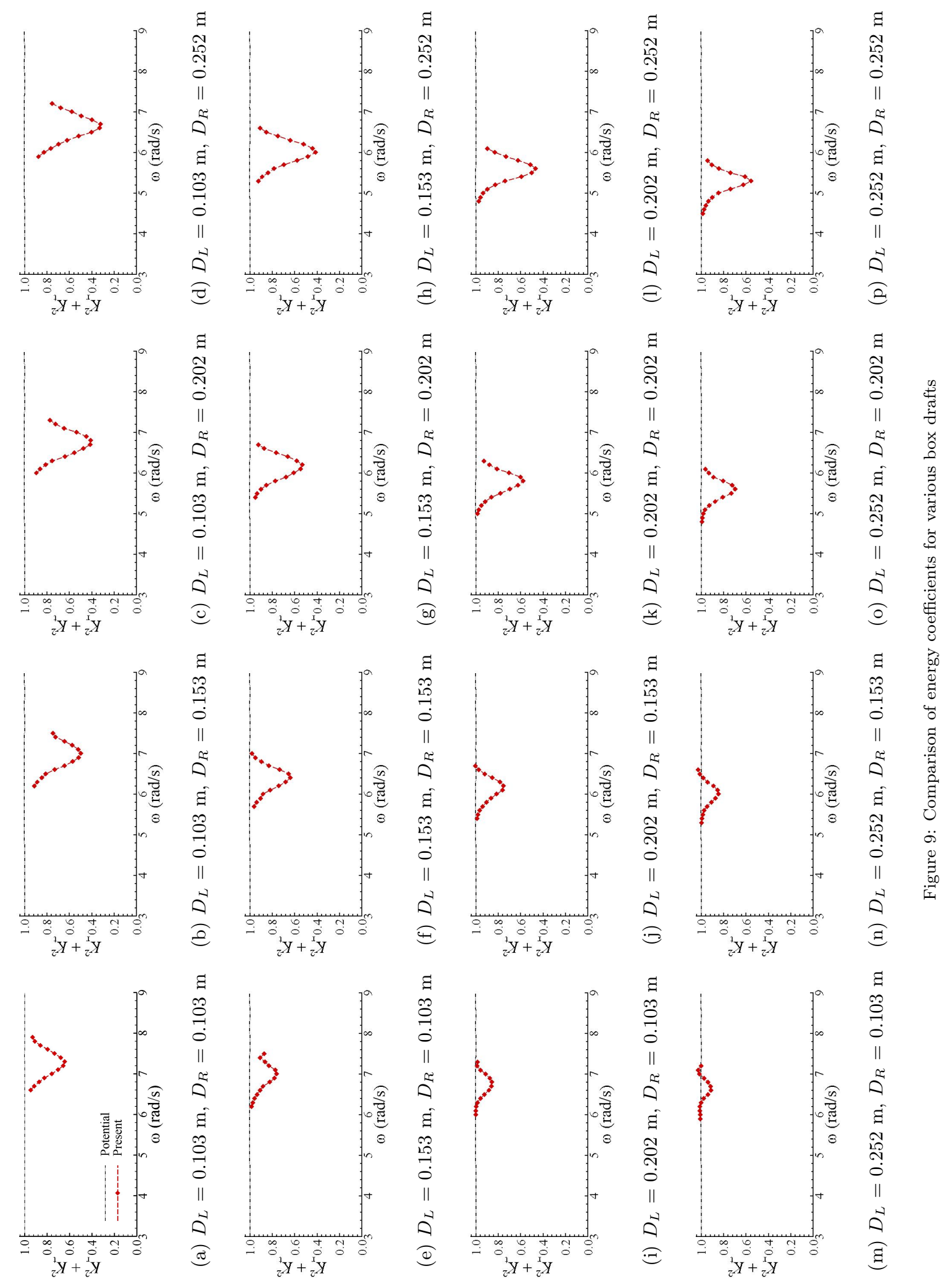


\subsection{Influence of downstream box draft}

The influence of downstream box draft on the wave response in the narrow gap is investigated in this section. As shown in Fig. 10 the variation of mean wave amplitude $A_{g} / A_{i}$ in the narrow gap with different downstream box drafts $D_{R}$ is examined. A general comparison suggests that the resonant frequency and amplitude tend to decrease and increase, respectively, with the increase of downstream box draft. It is believed that the large-amplitude piston-mode free surface oscillation in the narrow gap is essentially controlled by the volume of fluid entering into the narrow gap from the gap entrance. The volume of fluid entering into the narrow gap is dominated by the vertical velocity along the gap bottom. The dependence of wave amplitude in the narrow gap on the vertical velocity along the gap bottom has been reported in Lu et al. (2010), showing that the fluid flow in the vicinity of gap bottom has significant effect on wave amplitude in the narrow gap. Therefore, it is believed that the draft of upstream box is an important factor for the influence of downstream box draft on the wave amplitude in the narrow gap, which can be demonstrated in Fig. 10. As an example, in Fig. 10a, the draft of upstream box $D_{L}=0.103 \mathrm{~m}$ is the smallest one. With the increase of downstream box draft $D_{R}$, the position of gap bottom is kept on $0.103 \mathrm{~m}$. Increasing the downstream box draft $D_{R}$ can thus only enhance the wave action in the vicinity of the gap bottom. As for the case of $D_{L}=0.252 \mathrm{~m}$ in Fig. $10 \mathrm{~d}$. in which the draft of upstream box is the largest one, the position of gap bottom in fact also changes with the increase of downstream box draft $D_{R}$. This would certainly lead to more sensitive results of the variation of resonant response for various downstream box drafts in Fig. 10d,

Fig. 11 considers the influence of downstream box draft on the reflection and transmission coefficients around the resonant frequency. It can be seen that the frequency at which the minimal reflection coefficient occurs decreases with the increase of downstream box draft. Meanwhile, the insignificant variation of transmission coefficient by varying the downstream box draft around the resonant frequency can be observed, cf. Fig. 11. Correspondingly, the energy coefficient, $\mathbb{E}=K_{r}^{2}+K_{t}^{2}$, decreases with the increase of downstream box draft, as shown in Fig. 12 . By summarizing the results shown in Figs. 10-12, the following process of energy transformation and dissipation during the fluid resonant oscillation can be suggested. Firstly, the increase of downstream box draft tends to cause more large-amplitude piston-type of fluid oscillation. More energy dissipation will happen because the large-amplitude piston-type of fluid oscillation in the narrow gap can lead to significant fluid rotational motion. Moreover, the increase of fluid oscillation and energy dissipation is mainly dependent on the energy from the upstream box of the two-box system. It physically expresses the decrease of reflection coefficient with the increase of downstream box draft. As for the transmission wave, the downstream box draft has little effect in the present numerical results. 

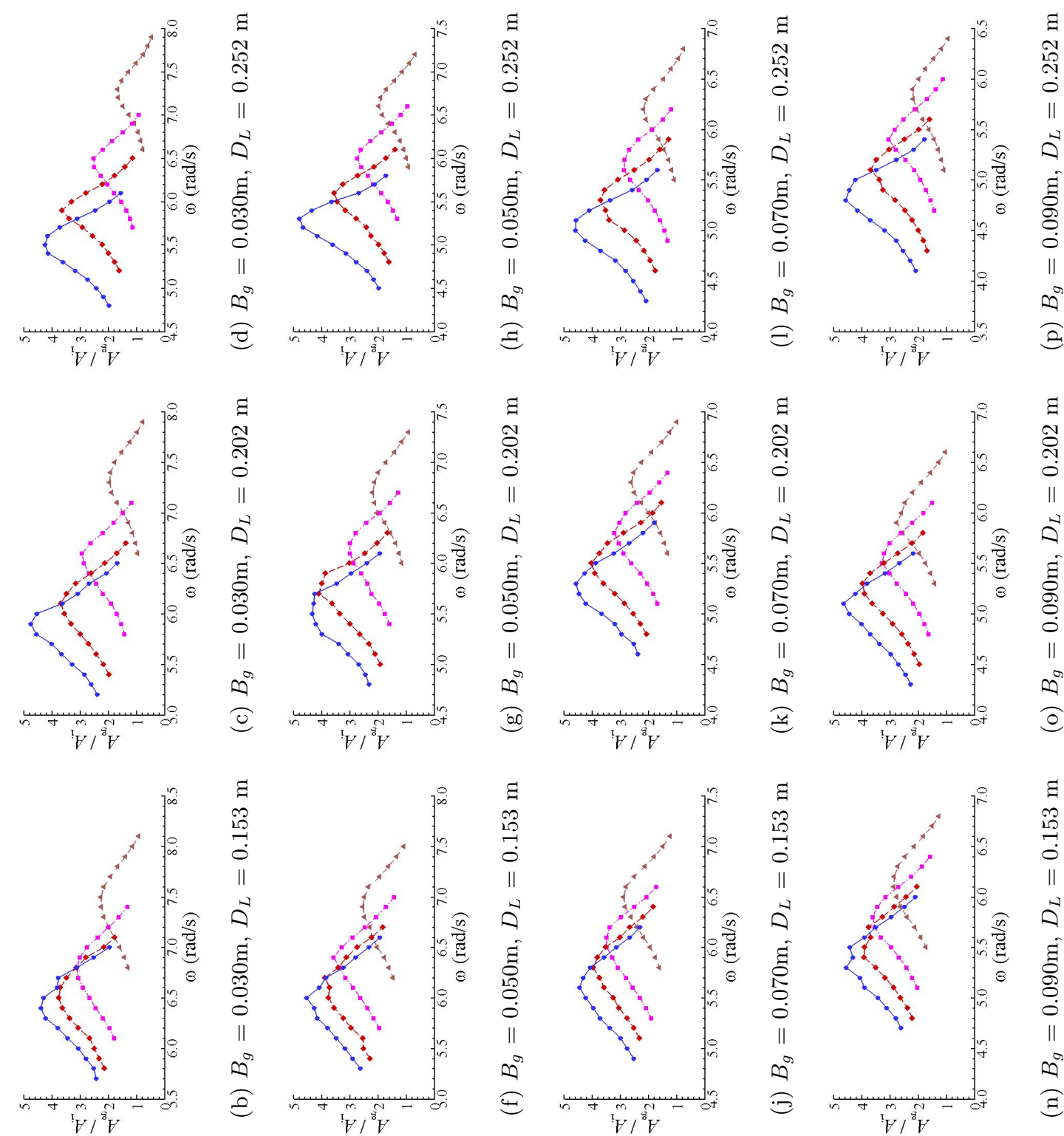

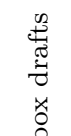
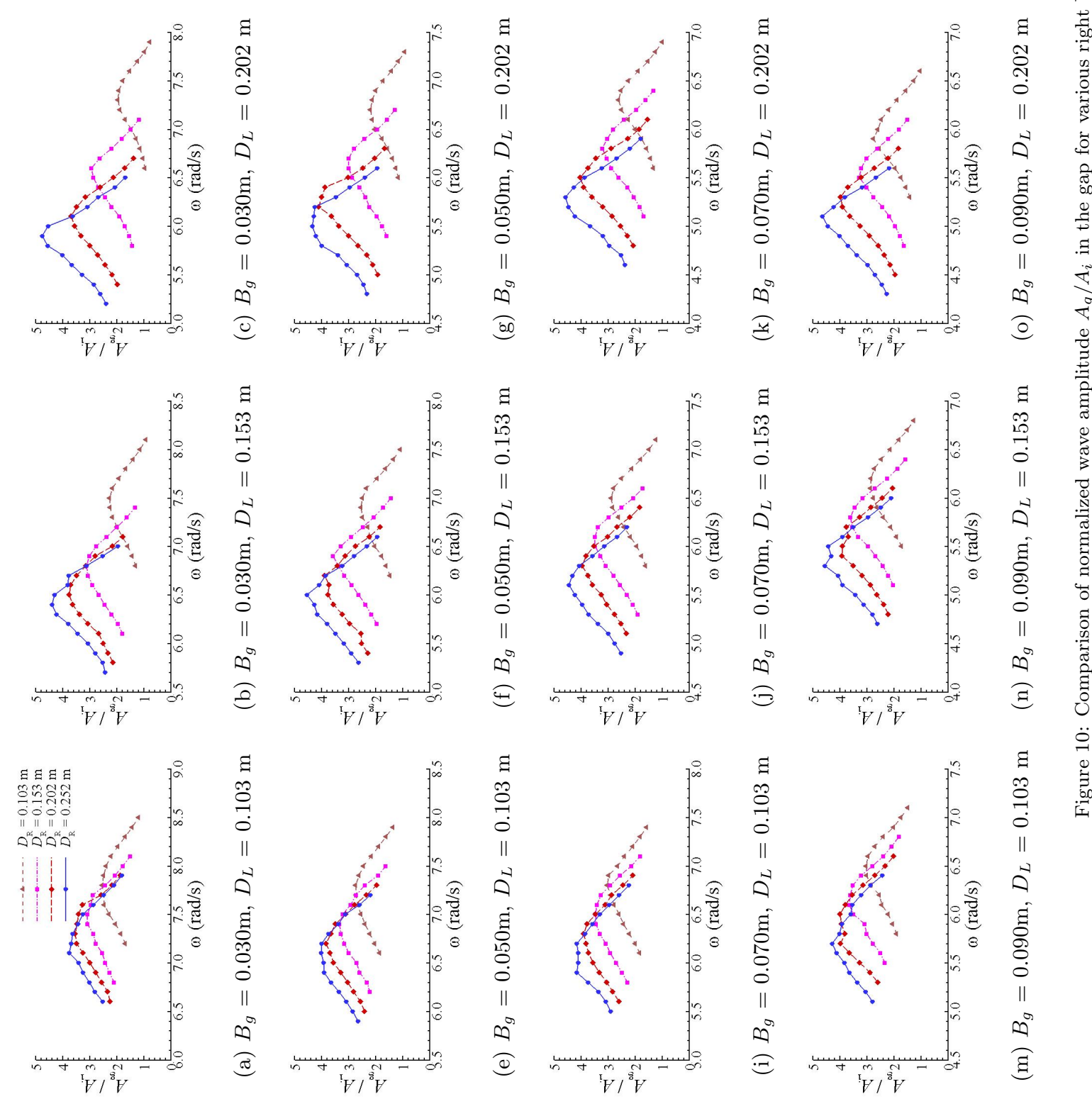

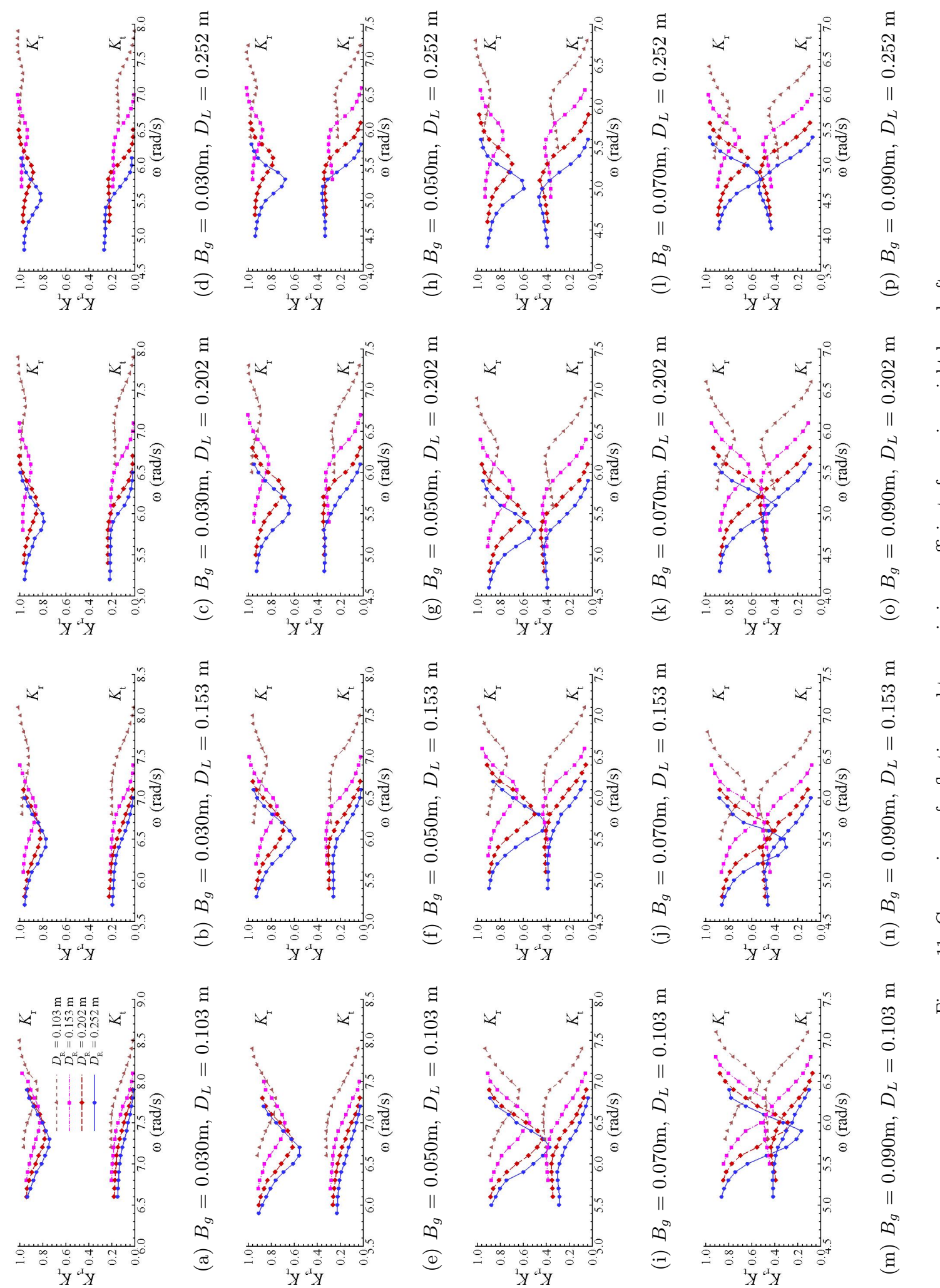

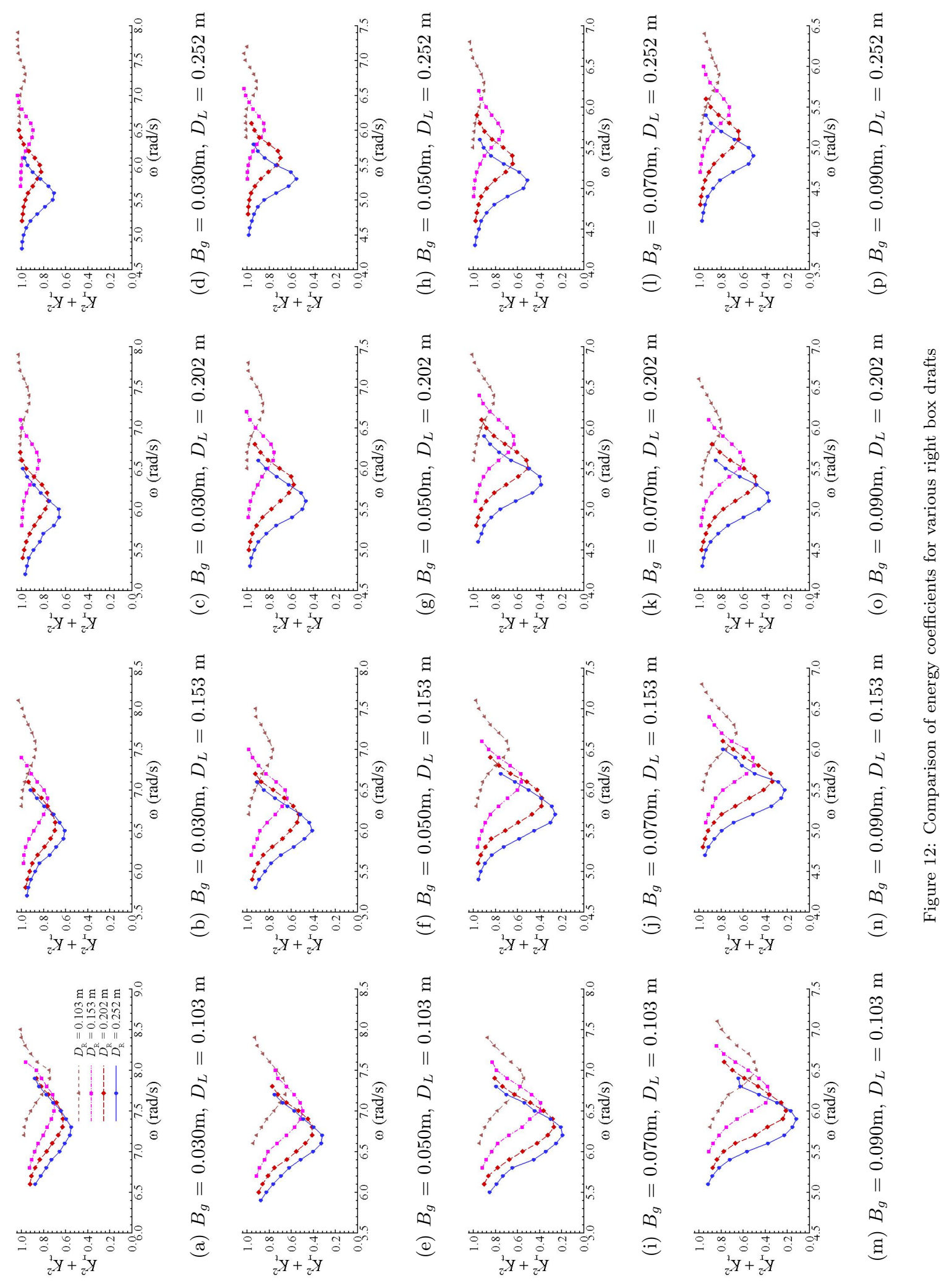


\subsection{Influence of upstream box draft}

Fig. 13 shows the influence of upstream box draft on the wave response in the narrow gap. A simple relation can be observed that the resonant frequency decreases with the increase of upstream box draft. Variation of resonant amplitude with different upstream box drafts is quite complex in these figures. When the draft of downstream box is smaller than that of upstream box, the gap bottom is shielded by the upstream box. It can be understood that the larger $D_{L}$ can lead to a stronger shielding effect on this occasion. Typical examples can be found in the first column of Fig. 13, where the draft of downstream box is the smallest one, $D_{R}=0.103 \mathrm{~m}$. In this situation, all the resonant amplitudes are observed to be smallest compared to those with the larger downstream box draft. When the draft of downstream box is larger than that of upstream box, the gap bottom is exposed to the incident wave action. In this case, the complex hydrodynamic behavior in the vicinity of the narrow gap is aroused due to the large-amplitude piston-type of resonant fluid oscillation, including the wave reflection from the leading wall of downstream box, the wave transformation below the bottom of downstream box, and the vortex shedding from the trailing sharp edge of upstream box. All these phenomena are not only relevant to the upstream box draft, but also closely dependent on the downstream box draft and gap breadth. Typical comparisons can be observed in the fourth column of Fig. 13 . As for the second and third columns of Fig. 13, combining the shielding effect and the direct wave action discussed above, more complex variation of resonant amplitude with different upstream box drafts can be observed.

The influence of upstream box draft on the reflection and transmission coefficients is illustrated in Fig. 14 The increase of upstream box draft can increase the reflection coefficients around the resonant frequency. In this case, more wave energy is reflected before entering into the two-box system. As for the transmission coefficient, again, the variation is insignificant compared to the reflection coefficient. In Fig. 15 the increase of energy coefficient with the increase of upstream box draft can be observed. Finally, comparison between Fig. 13 and Fig. 15 can also indicate that the large wave oscillations in the narrow gap do not always lead to the large energy dissipation, such as at the second, third and fourth columns in these figures. The reflection energy, expressed by the reflection coefficient, is also a significant parameter in this problem. 

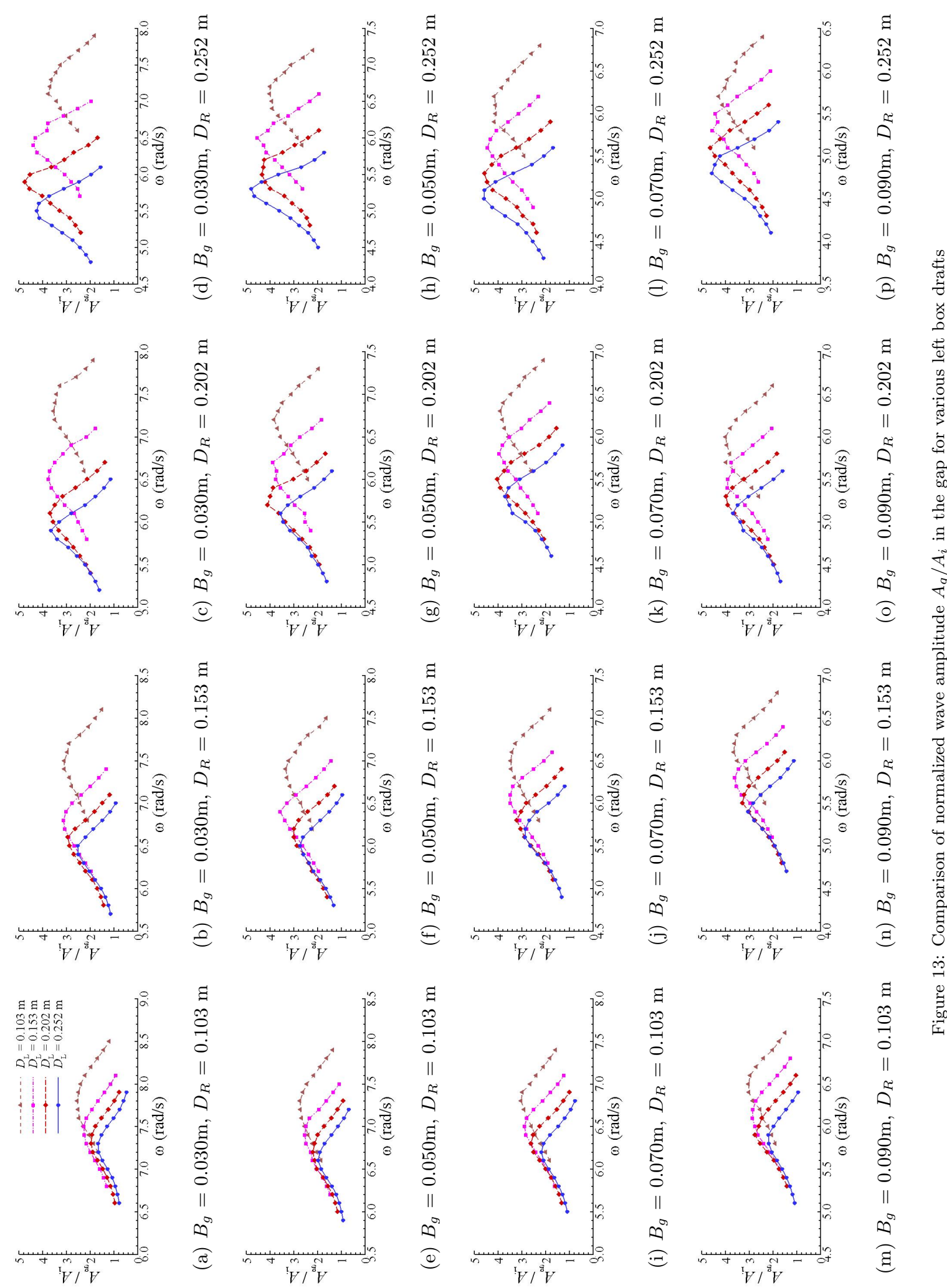

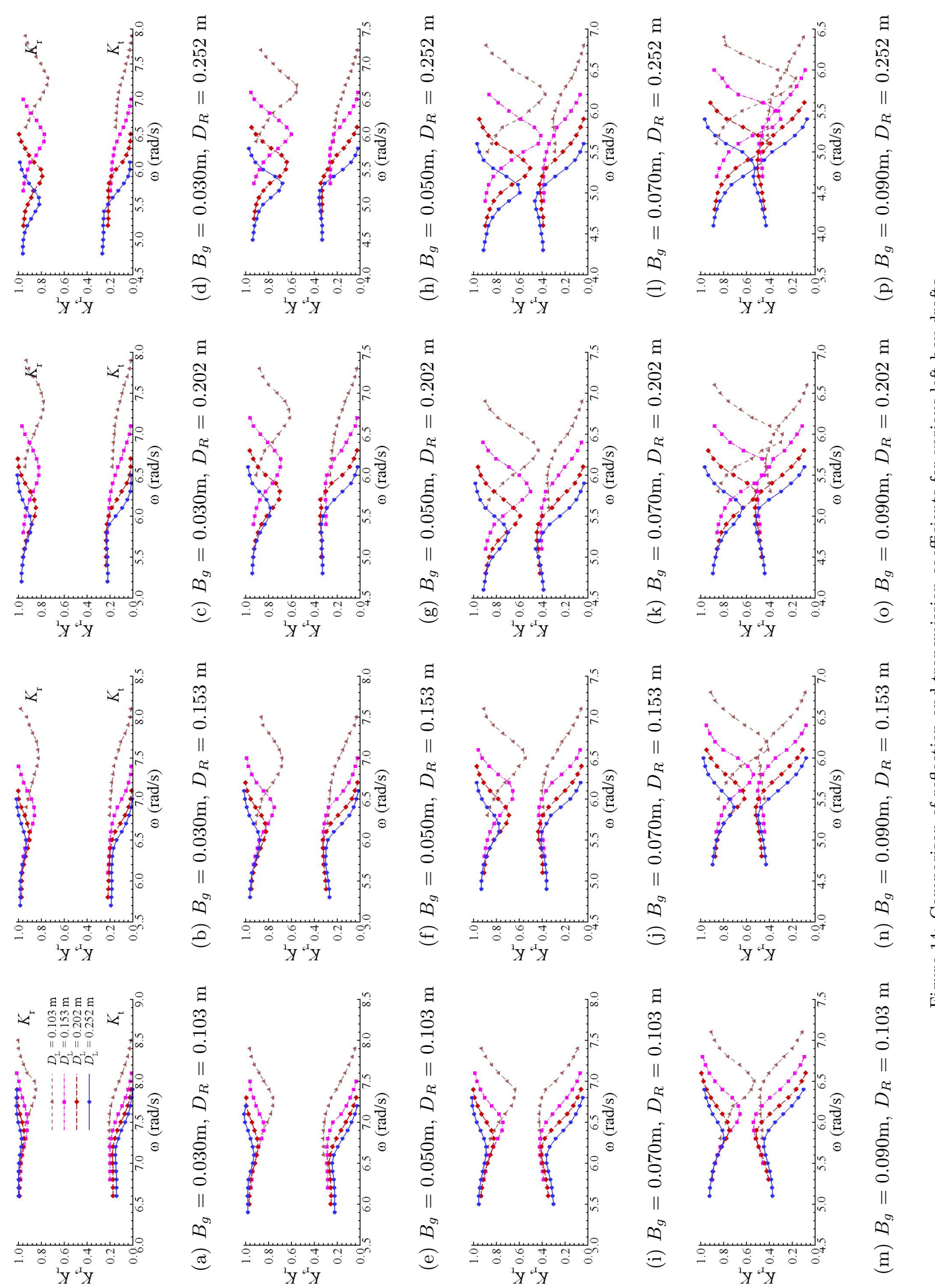

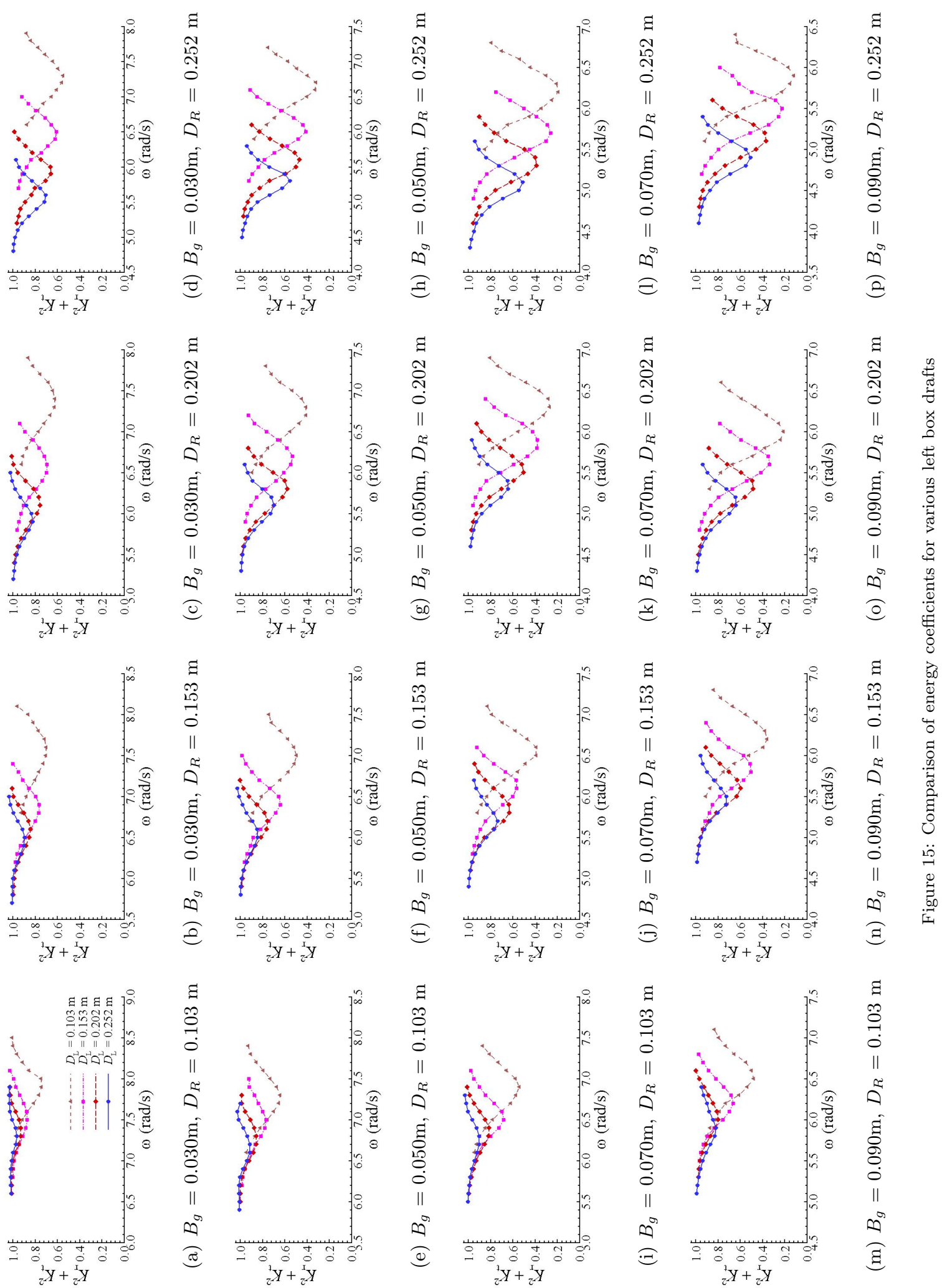


\subsection{Influence of gap breadth}

In order to clearly demonstrate the influence of gap breadth on the behavior of wave response in the narrow gap, the numerical results shown above are re-arranged in Fig. 16. It can be seen from these figures that for the specific drafts, larger gap breadth can cause smaller resonant frequency. As for the resonant wave amplitude in the narrow gap, it shows different characters with respect to the gap breadth with different upstream and downstream box drafts. That is, the effect of gap breadth is dependent on these two parameters. At the first column of Fig. 16, a general trend of the increase of resonant amplitude with the increase of gap breadth can be observed. Whereas, at the last column of Fig. 16, it seems hard to found a simple rule between the resonant amplitude and gap breadth. These phenomena are closely relevant to the wave reflection, transmission, and fluid rotational motion in the vicinity of the narrow gap.

The influence of gap breadth on reflection and transmission coefficients is considered, together with the energy coefficient. It can be seen from Fig. 17 that the reflection and transmission coefficients decrease and increase with the increase of gap breadth around the resonant frequency, respectively. Further analysis is carried out for the energy coefficient in Fig. 18, in which the energy coefficient decreases with the increase of gap breadth around the resonant frequency. According to the results shown in Figs. 16 - 18, it can be seen that with the increase of gap breadth, more fluid can be excited to be resonant in the narrow gap, and more energy is required to support the motion of this bulk fluid. On one hand, this energy comes from the incident wave, leading to the decrease of reflection coefficient. On the other hand, as a larger radiation source the oscillation of more fluid can cause the increase of transmission coefficient. It should be noted that more energy is dissipated due to the more fluid oscillation in the narrow gap. All these phenomena can also result in the complex hydrodynamic behavior of resonant wave amplitude with the variation of gap breadth. 

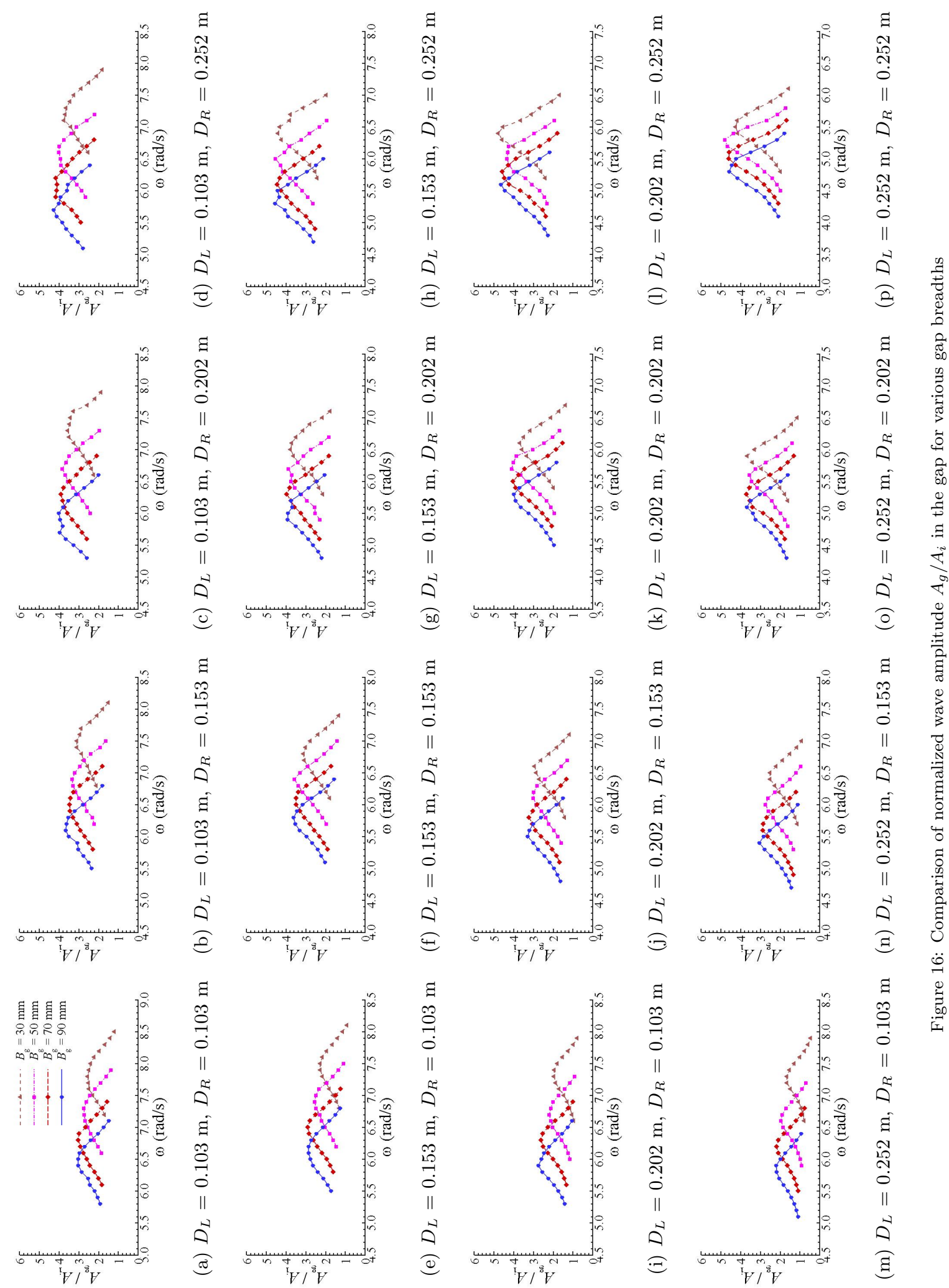


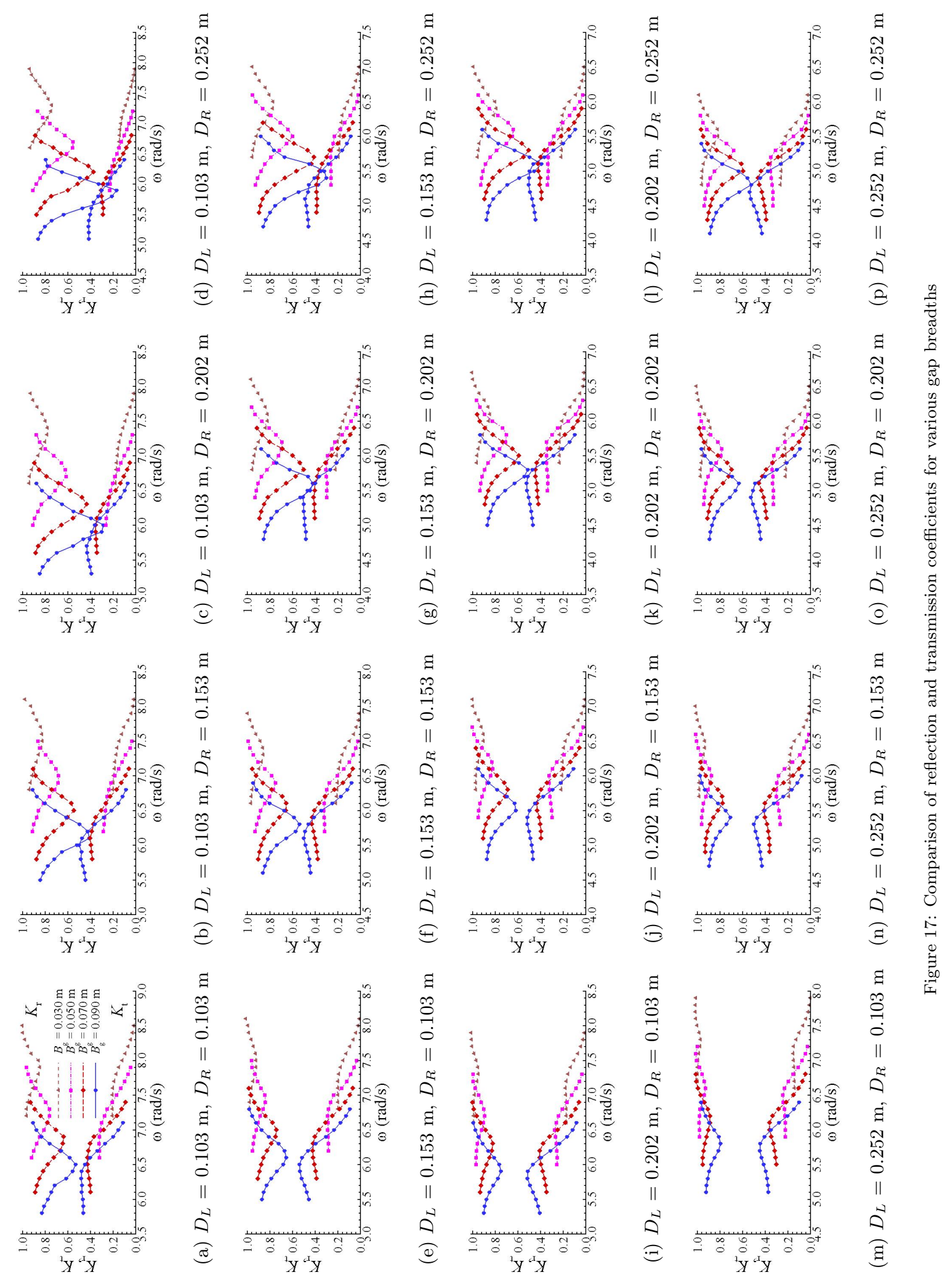



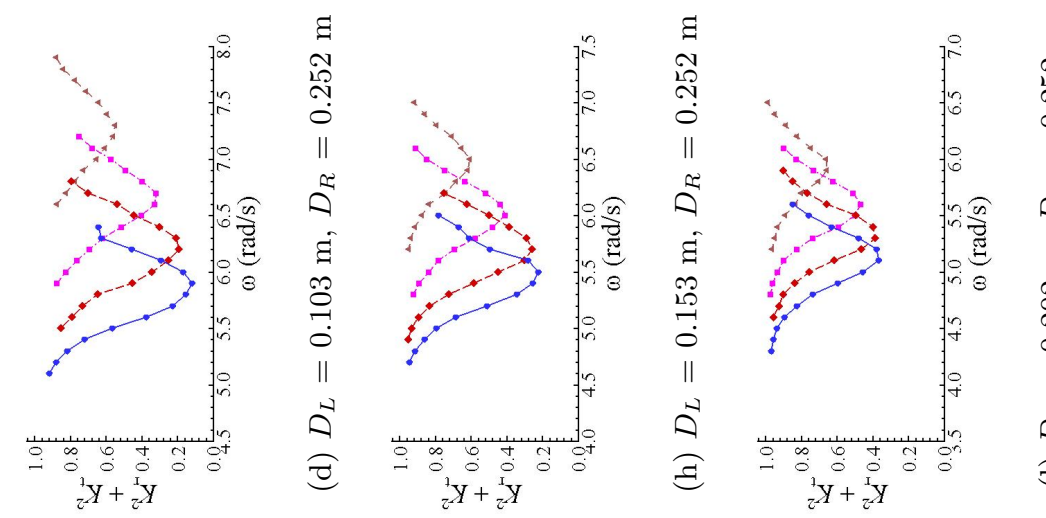

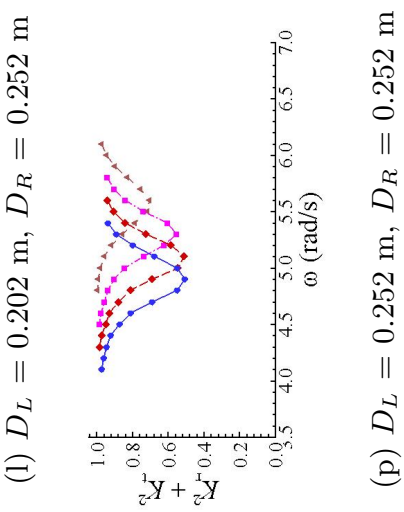
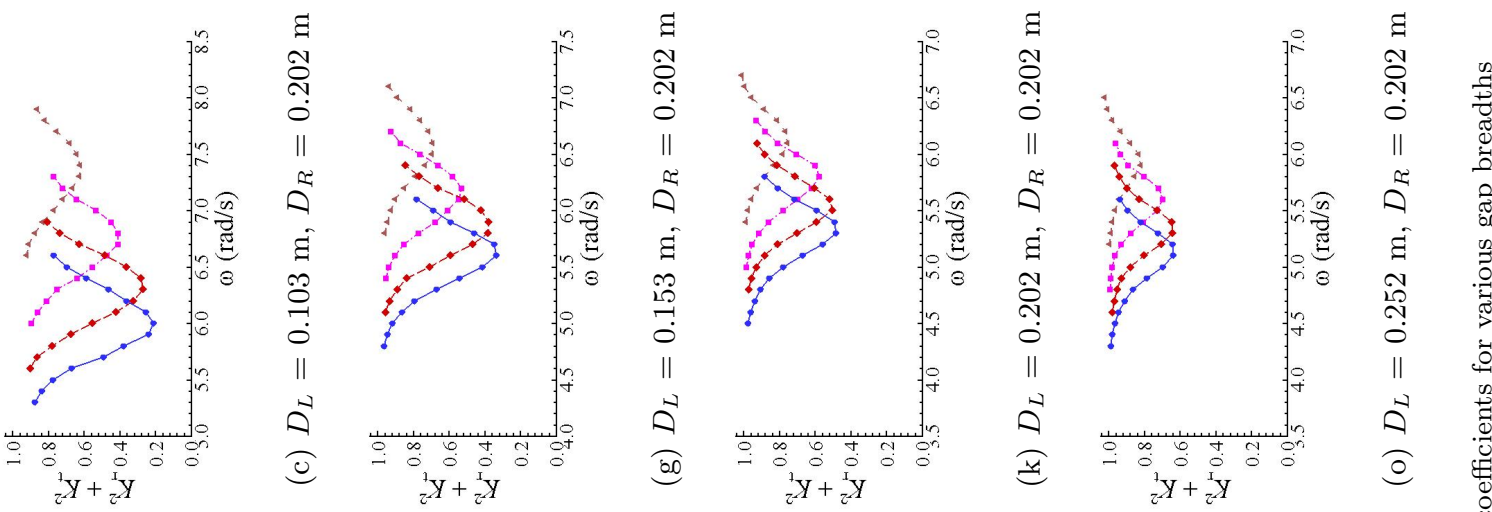

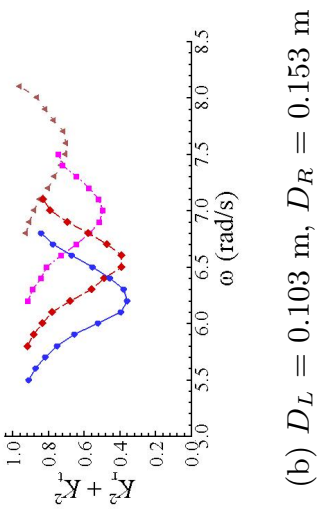
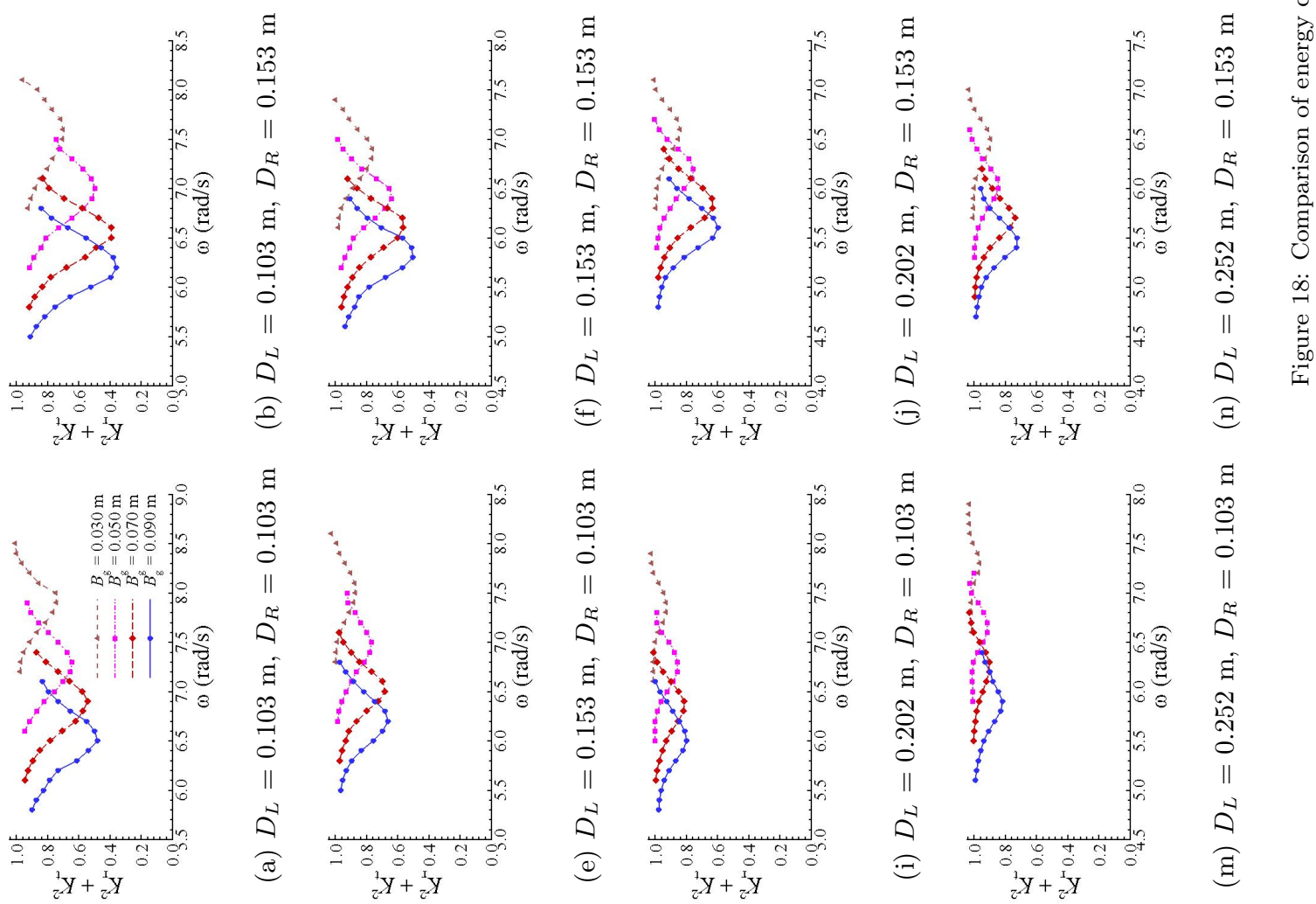


\subsection{Influence of incident wave amplitude}

Effect of incident wave amplitude on the wave response in the narrow gap is investigated in this section. Numerical results of $B_{g}=0.050 \mathrm{~m}$ with various box drafts at three different incident wave amplitudes, $A_{i}=$ $0.008 \mathrm{~m}, 0.012 \mathrm{~m}$ and $0.016 \mathrm{~m}$, are shown in Fig. 19. It can be seen that for a specific gap parameter, the wave response in the narrow gap decreases significantly with the increase of incident wave amplitude around the resonant frequency. However, if the incident wave frequency is far from the resonant frequency, little influence of incident wave amplitude on the wave response in the narrow gap can be observed. Numerical simulations also show a slight decrease in the resonant frequency with the increase of incident wave amplitude in Fig. 19 . More detailed comparisons illustrate that the large-amplitude piston-type of free surface oscillation and significant energy dissipation are relevant to the decrease of resonant frequency. For example, the frequency corresponding to the maximum amplitude is decreased from $\omega_{g}=6.6$ to $6.5 \mathrm{rad} / \mathrm{s}$ for Bg50DL103DR252 in Fig. $19 \mathrm{~d}$ as the incident wave amplitude increases from $A_{i}=0.008 \mathrm{~m}$ to $0.016 \mathrm{~m}$; while it keeps at $6.6 \mathrm{rad} / \mathrm{s}$ for Bg50DL252DR103 with those three incident wave amplitudes in Fig. $19 \mathrm{~m}$. The increase of mean water level in the narrow gap due to the free surface nonlinearity, the increase of added mass depending on the response wave amplitude, and larger damping ratio due to the energy dissipation are the three possible reasons for the decrease in the resonant frequency. The recent study in Faltinsen and Timokha (2015) quantified a pressure discharge condition in the gap opening, where a nonlinear integral term was derived in the dynamic condition on gap surface. It may be fit for further describing the slight decrease of resonant frequency, as well as measuring the energy dissipation in the vicinity of the gap.

The influence of incident wave amplitude on the behavior of reflection and transmission coefficients, $K_{r}$ and $K_{t}$, is considered in Fig. 20. Numerical results suggest that the transmission coefficients decrease with the increase of incident wave amplitude at the resonant frequency, implying that less percent of energy is transmitted to the downward of the two-body system. On the other hand, the reflection coefficients increase with the increase of incident wave amplitude at the resonant frequency. Generally, the sensitivity of reflection coefficient to the variation of incident wave amplitude is stronger than that of transmission coefficient. It in fact leads to the results in Fig. 21, in which the energy coefficient increases with the increase of incident wave amplitude at the resonant frequency. In other words, relatively less energy dissipation happens for the large incident wave amplitude at the resonant frequency. All these phenomena indicate that the increase of incident wave amplitude with the larger wave amplitude tends to enlarge the reflection energy, and hence relatively less wave energy can enter into the narrow gap. Therefore, smaller resonant wave response in the narrow gap and less energy dissipation can be found at the resonant frequency. The analysis demonstrates that the energy transformation due to the wave reflection is one determinative factor for the decrease of resonant wave response with the increase of incident wave amplitude at the resonant frequency. Closer comparisons in Figs. 20 and 21 indicate that the smaller reflection and energy coefficients can be obtained with the larger incident wave amplitudes at a frequency slightly away from the resonant one, which implies that the relative energy dissipation due to the fluid rotational motion becomes the dominant factor in this situation. In sum, 
${ }_{428}$ both the energy transformation and the energy dissipation play the important, but different roles for fluid 429 resonances in the narrow gap. 

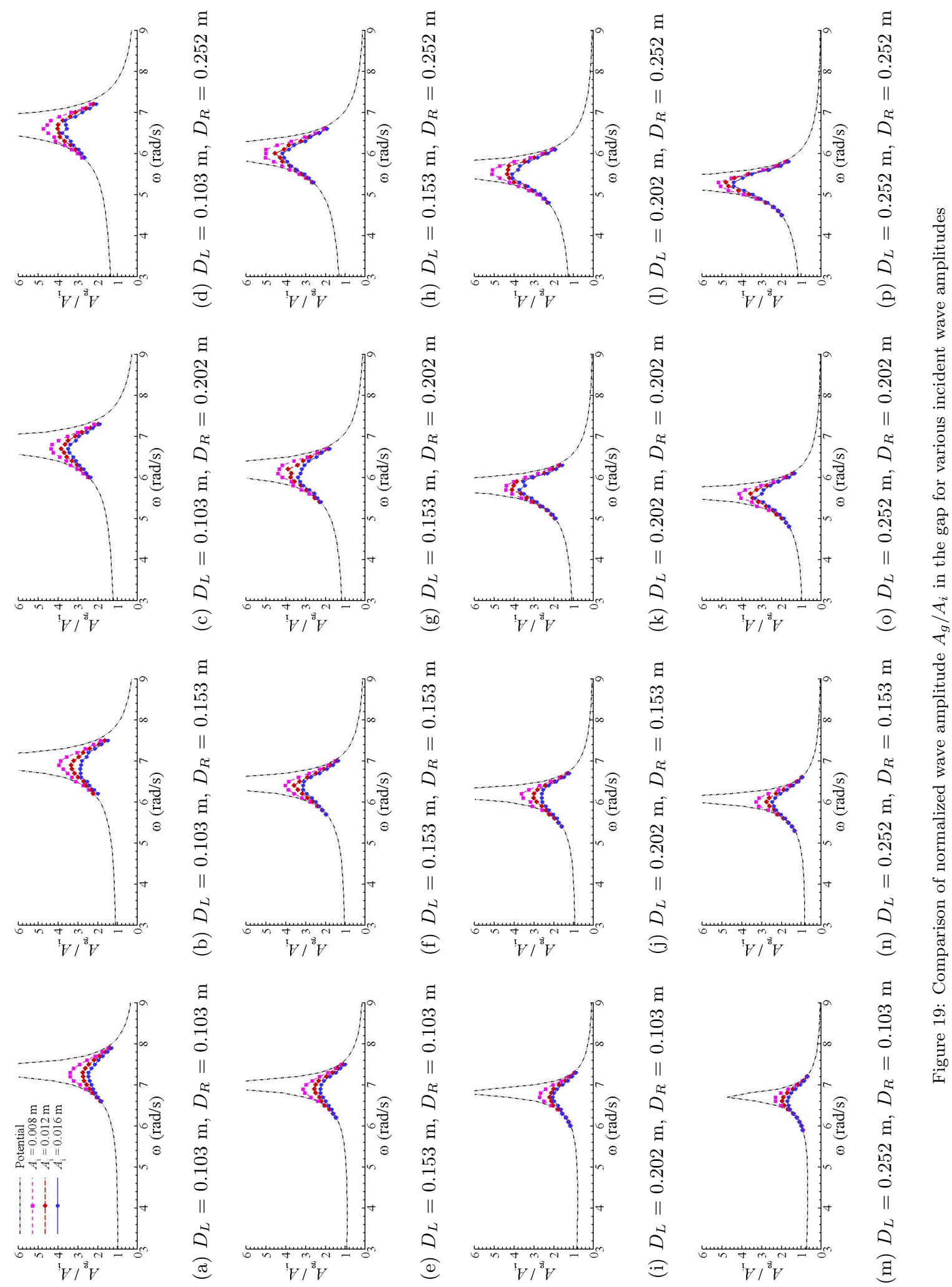

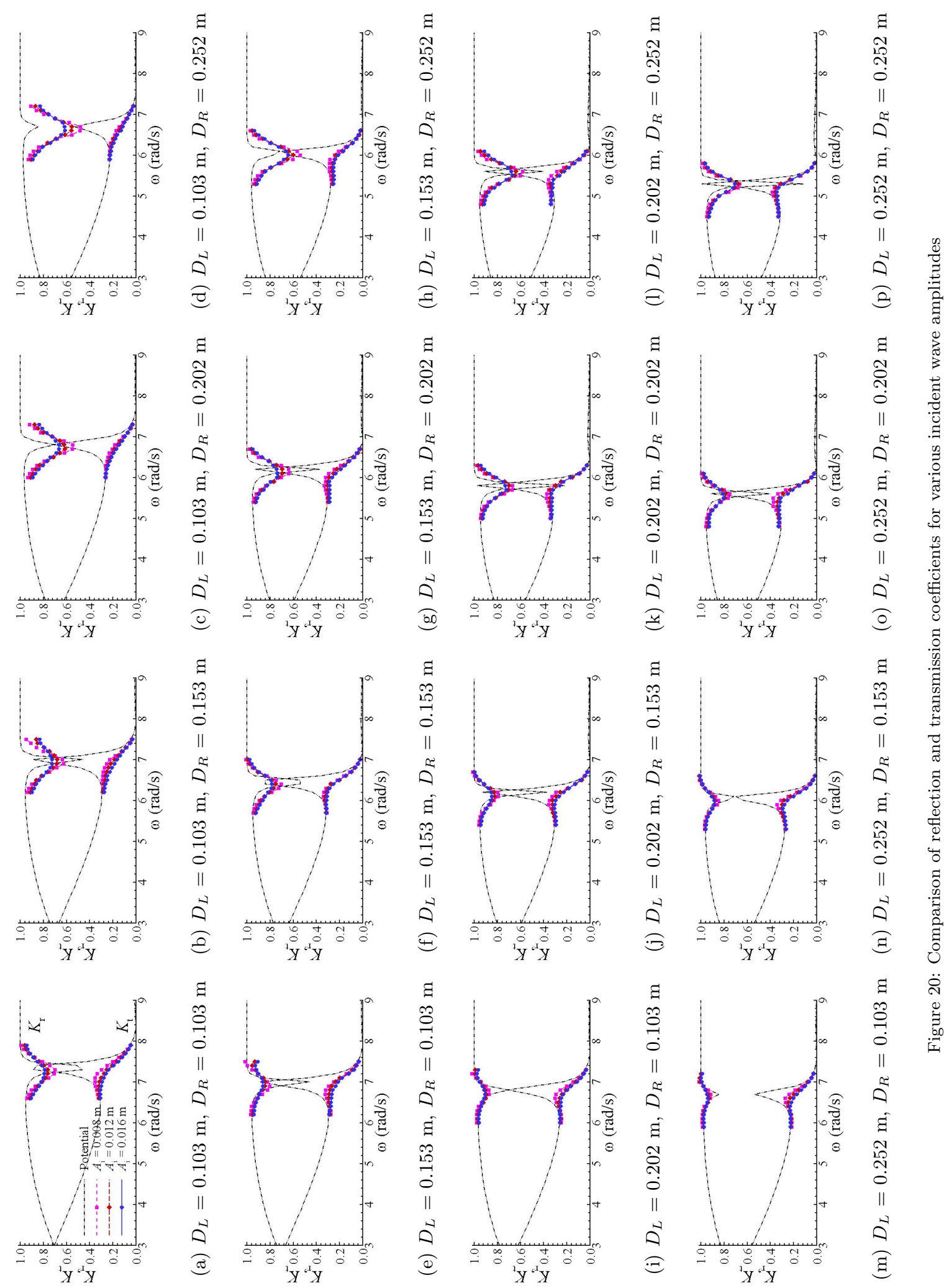

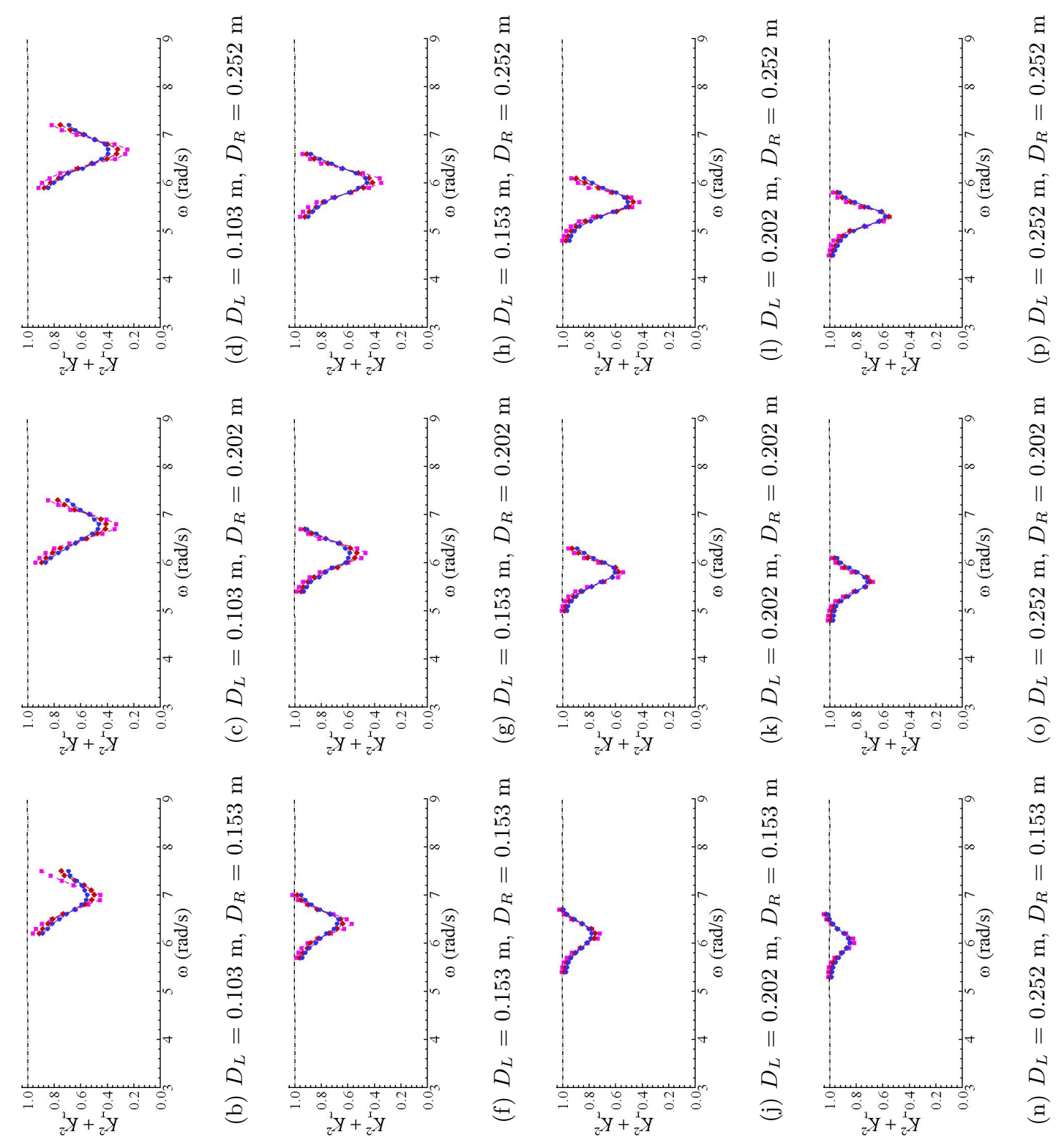

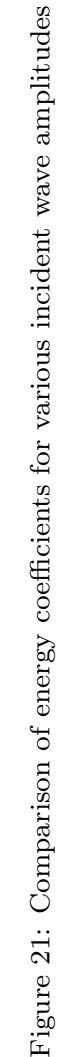
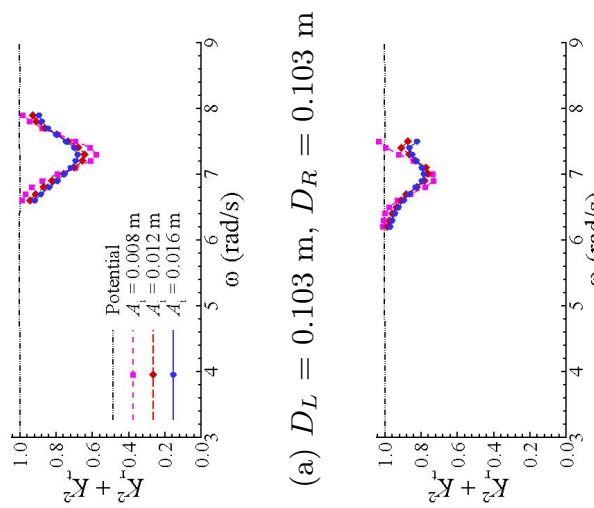

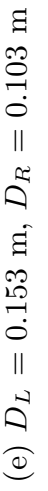
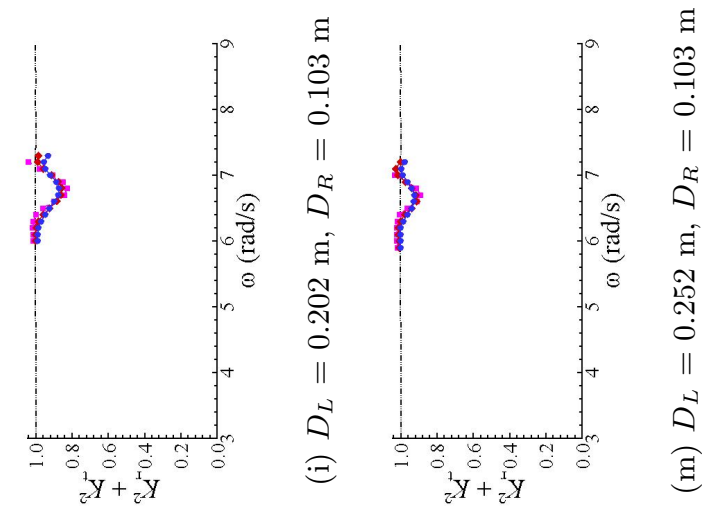


\section{Conclusion}

The Navier-Stokes equations are adopted for investigating the hydrodynamic behaviours of fluid resonance in the narrow gap formed by two side-by-side non-identical boxes. In order to obtain a clear understanding of the influence of gap configurations and wave conditions on the wave response, three different incident wave amplitudes with different frequencies are considered at different gap breadths, upstream and downstream box drafts, comprising in total 192 different cases. Numerical investigations include the wave amplitude in the narrow gap, the reflection and transmission coefficients, $K_{r}$ and $K_{t}$, and the energy coefficient, defined as $\mathbb{E}=K_{r}^{2}+K_{t}^{2}$. The mechanical essence of the gap resonance between two non-identical boxes is explained from the perspective of energy transformation and energy dissipation. The main findings are illustrated as follows:

1) Consistent with the theoretical analysis, the numerical simulations suggest that the resonant frequency tends to be smaller with the increase of gap breadth, upstream and downstream box drafts. The incident wave steepness hardly affects the resonant frequency; only a slight decrease of the resonant frequency can be observed with the increase of incident wave amplitude for the cases with larger energy dissipation.

2) The draft of downstream box has the significant influence on the wave response around the resonant frequency. With the increase of downstream box draft, the increase of wave response and energy dissipation can be observed, which is mainly supported by the energy from the upstream box of the two-box system. It physically expresses the decrease of reflection coefficient with the increase of downstream box draft.

3) When the draft of downstream box is smaller than that of upstream box, stronger shielding effect can be observed with the increase of upstream box draft. If the draft of downstream box is larger than that of upstream box, the increasing reflection coefficient and energy coefficient can be observed with the increase of upstream box draft, because of the enhanced wave reflection.

4) With the increase of gap breadth, more energy is required to excite the large-amplitude piston-type of fluid oscillation as more resonant fluid exists in the narrow gap, which leads to the decrease of reflection coefficient, and the increase of transmission coefficient. More energy dissipation also happens due to the fact that more fluid oscillates in the narrow gap with the increase of gap breadth.

5) For the small upstream and large downstream box drafts, the energy dissipation in the narrow gap is dominant, resulting in that the wave response in the narrow gap by viscous flow model is smaller than that by potential flow model. As for the large upstream and small downstream box drafts, the wave response in the narrow gap by the viscous flow model are always larger than that by the linear potential flow model because more wave energy is reflected before entering into the narrow gap. If the difference between the upstream and downstream box drafts is not large, a combining hydrodynamic behavior of the previous two typical cases can be observed.

6) As increasing the incident wave amplitude, the reflection coefficient becomes larger at the resonant frequency, which leads to the decrease of transmission coefficient, relative wave response in the narrow gap, as well as energy dissipation at the resonant frequency. The energy transformation due to the 
large-amplitude free surface motion can not be ignored in this situation. When away from the resonant frequency, smaller reflection coefficient and energy coefficient can be obtained, implying that the energy dissipation becomes the dominant factor for the decrease of wave response in the narrow gap with the increase of incident wave amplitude.

\section{Acknowledgement}

This work was supported by the Natural Science Foundation of China with Grant No. 51490673. The financial support from the Petro China Innovation Foundation with contract No. 2016D-5007-0601 is also acknowledged. The authors acknowledge the Supercomputer Center of Dalian University of Technology for providing computing resources.

\section{References}

Ananthakrishnan, P. (2015). Viscosity and nonlinearity effects on the forces and waves generated by a floating twin hull under heave oscillation, Applied Ocean Research 51: 138-152.

Berberović, E., van Hinsberg, N. P., Jakirlić, S., Roisman, I. V. and Tropea, C. (2009). Drop impact onto a liquid layer of finite thickness: Dynamics of the cavity evolution, Physical Review E 79(3): 036306.

Chen, X.-B. (2004). Hydrodynamics in offshore and naval applications, Keynote lecture in the 6th International conference of Hydrodynamics, Perth, Australia.

Elie, B., Reliquet, G., Guillerm, P.-E., Thilleul, O., Ferrant, P., Gentaz, L. and Ledoux, A. (2013). Simulation of the gap resonance between two rectangular barges in regular waves by a free surface viscous flow solver, The 32nd International Conference on Ocean, Offshore and Arctic Engineering (OMAE), Nantes, France.

Engsig-Karup, A. P. (2006). Unstructured nodal DG-FEM solution of high-order boussinesq-type equations, $\mathrm{PhD}$ thesis, Technical University of Denmark.

Faltinsen, O. M., Rognebakke, O. F. and Timokha, A. N. (2007). Two-dimensional resonant piston-like sloshing in a moonpool, Journal of Fluid Mechanics 575: 359-397.

Faltinsen, O. and Timokha, A. (2015). On damping of two-dimensional piston-mode sloshing in a rectangular moonpool under forced heave motions, Journal of Fluid Mechanics 772: R1.

Feng, X. and Bai, W. (2015). Wave resonances in a narrow gap between two barges using fully nonlinear numerical simulation, Applied Ocean Research 50: 119-129.

Fredriksen, A. G., Kristiansen, T. and Faltinsen, O. M. (2014). Experimental and numerical investigation of wave resonance in moonpools at low forward speed, Applied Ocean Research 47: 28-46. 
Fuhrman, D. R., Madsen, P. A. and Bingham, H. B. (2006). Numerical simulation of lowest-order shortcrested wave instabilities, Journal of Fluid Mechanics 563: 415-441.

Hirt, C. W. and Nichols, B. D. (1981). Volume of fluid (vof) method for the dynamics of free boundaries, Journal of computational physics 39(1): 201-225.

Issa, R. I. (1986). Solution of the implicitly discretised fluid flow equations by operator-splitting, Journal of computational physics 62(1): 40-65.

Iwata, H., Saitoh, T. and Miao, G. (2007). Fluid resonance in narrow gaps of very large floating structure composed of rectangular modules, Proceedings of the Fourth International Conference on Asian and Pacific Coasts, Nanjing, China, pp. 815-826.

Jacobsen, N. G., Fuhrman, D. R. and Fredsøe, J. (2012). A wave generation toolbox for the open-source cfd library: Openfoam®, International Journal for Numerical Methods in Fluids 70(9): 1073-1088.

Jasak, H. (1996). Error analysis and estimation for the finite volume method with applications to fluid flows, $\mathrm{PhD}$ thesis, Imperial College London (University of London).

Jean-Robert, F., Naciri, M. and Chen, X.-B. (2006). Hydrodynamics of two side-by-side vessels experiments and numerical simulations, The Sixteenth International Offshore and Polar Engineering Conference, San Francisco, USA.

Kristiansen, T. and Faltinsen, O. M. (2010). A two-dimensional numerical and experimental study of resonant coupled ship and piston-mode motion, Applied Ocean Research 32(2): 158-176.

Kristiansen, T. and Faltinsen, O. M. (2012). Gap resonance analyzed by a new domain-decomposition method combining potential and viscous flow draft, Applied Ocean Research 34: 198-208.

Lu, L. and Chen, X.-B. (2012). Dissipation in the gap resonance between two bodies, The 27th International Workshop on Water Waves and Floating Bodies, Copenhagen, Denmark.

Lu, L., Cheng, L., Teng, B. and Zhao, M. (2010). Numerical investigation of fluid resonance in two narrow gaps of three identical rectangular structures, Applied Ocean Research 32(2): 177-190.

Lu, L., Teng, B., Cheng, L., Sun, L. and Chen, X. (2011). Modelling of multi-bodies in close proximity under water waves-fluid resonance in narrow gaps, Science China Physics, Mechanics and Astronomy 54(1): $16-25$.

Lu, L., Teng, B., Sun, L. and Chen, B. (2011). Modelling of multi-bodies in close proximity under water waves-fluid forces on floating bodies, Ocean Engineering 38(13): 1403-1416.

Mayer, S., Garapon, A., Sørensen, L. et al. (1998). A fractional step method for unsteady free-surface flow with applications to non-linear wave dynamics, International Journal for Numerical Methods in Fluids 28(2): 293-315. 
Molin, B. (2001). On the piston and sloshing modes in moonpools, Journal of Fluid Mechanics 430: 27-50.

Molin, B., Remy, F., Kimmoun, O. and Stassen, Y. (2002). Experimental study of the wave propagation and decay in a channel through a rigid ice-sheet, Applied ocean research 24(5): 247-260.

Moradi, N., Zhou, T. and Cheng, L. (2016). Two-dimensional numerical study on the effect of water depth on resonance behaviour of the fluid trapped between two side-by-side bodies, Applied Ocean Research 58: $218-231$.

Newman, J. (2004). Progress in wave load computations on offshore structures, Invited Lecture in The 23rd International Conference on Ocean, Offshore and Arctic Engineering (OMAE), Vancouver, Canada.

Rusche, H. (2003). Computational fluid dynamics of dispersed two-phase flows at high phase fractions, PhD thesis, Imperial College London (University of London).

Saitoh, T. (2007). Private communication.

Saitoh, T., Miao, G. and Ishida, H. (2006). Theoretical analysis on appearance condition of fluid resonance in a narrow gap between two modules of very large floating structure, Proceedings of the Third Asia-Pacific Workshop on Marine Hydrodynamics, Shanghai, China, pp. 170-175.

Sun, L., Eatock Taylor, R. and Taylor, P. H. (2010). First-and second-order analysis of resonant waves between adjacent barges, Journal of Fluids and Structures 26(6): 954-978. 